KANAZAWA-07-18

\title{
Flavor Changing Neutral Higgs Bosons in a Supersymmetric Extension based on a $Q_{6}$ Family Symmetry
}

\author{
Naoko Kifune ${ }^{a}$, Jisuke $\mathrm{Kubo}^{a}$, and Alexander Lenz ${ }^{b}$ \\ a Institute for Theoretical Physics, Kanazawa University, Kanazawa 920-1192, Japan \\ b Universität Regensburg, D-93051 Regensburg, Germany
}

\begin{abstract}
A supersymmetric extension of the standard model based on the discrete $Q_{6}$ family symmetry is considered, and we investigate flavor-changing neutral current (FCNC) processes, especially those mediated by heavy flavor-changing neutral Higgs bosons. Because of the family symmetry the number of the independent Yukawa couplings is smaller than that of the observed quantities such as the Cabibbo-Kobayashi-Maskawa matrix and the quark masses, so that the FCNCs can be parametrized only by the mixing angles and masses of the Higgs fields. We focus our attention on the mass differences of the neutral $K, D$ and $B$ mesons. All the constraints including that from the ratio $\Delta M_{B_{s}} / \Delta M_{B_{d}}$ can be satisfied, if the heavy Higgs bosons are heavier than $\sim 1.5 \mathrm{TeV}$. If the constraint from $\Delta M_{K}$ is slightly relaxed, the heavy Higgs bosons can be as light as $\sim 0.4 \mathrm{TeV}$, which is within the accessible range of LHC.
\end{abstract}

PACS numbers: 11.30.Hv, 12.15.Ff ,12.60.Jv,14.80.Cp 


\section{INTRODUCTION}

In recent studies on flavor symmetries ${ }^{1}$ it has become clear that a flavor symmetry can be realized at low energies. As long as this possibility is not excluded, theoretical as well as experimental searches for a low energy flavor symmetry should be continued. An important prediction of any viable low energy flavor symmetry, which is broken only spontaneously or at most softly, is the existence of multiple $S U(2)_{L}$ doublet Higgs fields, as one could read off from a sort of no-go theorem of [4]. This implies that there should exist several neutral Higgs fields that have flavor changing couplings to the fermions at the tree-level. Therefore, an observation of a non-standard flavor changing neutral current (FCNC) process, at LHC for instance, is not necessarily an indication of supersymmetry [5, 6].

In Ref. [7] a supersymmetric flavor model based on a dicyclic dihedral group $Q_{6}$ has been suggested. ${ }^{2}$ The main motivation there was to derive a modified Fritzsch mass matrix for the quarks from a flavor symmetry. With an assumption that $\mathrm{CP}$ is spontaneously broken, the model can fix six quark masses and four Cabibbo-Kobayashi-Maskawa (CKM) parameters in term of nine parameters of the model. It has been later realized in Refs. [21, 22] that through an appropriate change of the lepton assignment the leptonic sector can be brought into the same form as that of the model of [23, 24]. Then there are only seven parameters in the leptonic sector of the model to fix six lepton masses and six Maki-Nakagawa-Sakata (MNS) parameters. The discrete flavor group $Q_{6}$ is the smallest non-abelian group with which the above situation can be achieved.

However, it turned out that one has to introduce a certain set of $S U(2)_{L} \times U(1)_{Y}$ singlet fields and also additional abelian global symmetries to make the model viable. Nothing is wrong with this situation, but in this paper we would like to stress the minimal content of the Higgs fields and at the same time a "one + two" structure for each family; one $Q_{6}$ singlet and one $Q_{6}$ doublet for each family including the $S U(2)_{L}$ doublet Higgs fields. In Sec. II we will shed light upon the relation between the non-renormalization theorem and flavor symmetry, and will show that different flavor symmetries can be consistently introduced into a softly broken supersymmetric gauge theory. We will systematically investigate this possibility in a general framework. With this observation we will find in Sec. III that the one + two structure of family in a minimal $Q_{6}$ extension of the supersymmetric standard model (MSSM) can be consistently realized.

In Sec. IV we will consider the Higgs sector. Because of the one + two structure the Higgs sector is much simpler than that of [7, 21, 22], and therefore the sector can be investigated

\footnotetext{
${ }^{1}$ For recent reviews see, for instance, [1, 2, 3].

${ }^{2} Q_{6}$ is one of $Q_{2 N}$ with $N=2,3, \ldots$, which are the "covering groups" of the dihedral groups $D_{N}[8,9]$. In recent years there are a number of interesting flavor models based on $Q_{2 N}$ and $D_{N}$. For instance, $D_{4}$ has been used as a flavor symmetry in Refs. [10, 11, 12, 13, 14], while $D_{5}, D_{6}, D_{7}$ and $Q_{4}$ have been considered in Refs. [15], 16], [17] and [18], respectively. See also Refs. 19, 20].
} 
with much less assumptions. We will explicitly show that it is possible to fine tune the soft-supersymmetry-breaking (SSB) parameters so as to make the heavy Higgs bosons much heavier (several $\mathrm{TeV}$ ) than $M_{Z}$ and at the same time to obtain a desired size of spontaneous $\mathrm{CP}$ violation to reproduce the Kobayashi-Maskawa CP violating phase.

In Sec. $\mathrm{V}$ we will first calculate the unitary matrices that diagonalize the fermion mass matrices, which are needed to write down the Yukawa couplings in terms of mass eigenstates. We only briefly mention FCNCs and CP violations in the SSB sector and in the lepton sector, because detailed investigations on these subjects have been recently carried out in Ref. [22] and in Ref. [25], respectively. Instead we investigate FCNC processes mediated by neutral heavy Higgs fields. We concentrate on the constraints coming from the mass differences in the neutral meson systems, $\Delta M_{K}, \Delta M_{B_{s}}, \Delta M_{B_{d}}$ and $\Delta M_{D}$, in a similar spirit as Refs. [26, 27, 28, 29, 30, 31, 32] and references therein. We express the relevant flavor changing-neutral Yukawa couplings in terms of the mass eigenstates, where except the phases the size of the Yukawa couplings are basically fixed. Allowed ranges in which the constraints are satisfied are shown in different figures. We find that the heavy Higgs bosons should be heavier than $\sim 1.5 \mathrm{TeV}$, although it is possible to fine tune the parameters such that the constraints can be satisfied for lighter mass values.

Sect. VI is devoted for conclusion.

\section{NON-RENORMALIZATION THEOREM AND FLAVOR SYMMETRY}

A flavor symmetry can control the structure of the independent parameters of a theory. In supersymmetric theories, moreover, the non-renormalization theorem allows to suppress certain couplings and also to relate them with each other, without facing contradictions with renormalization. What is therefore the (technical) role of a flavor symmetry in supersymmetric theories? We recall that the D-terms are renormalized and the wave function renormalization can mix matter superfields $\Phi_{i}$ 's in general. Therefore, starting with diagonal kinetic terms $\Phi_{i}^{*} \Phi_{i}$ is not always consistent with renormalization. If a non-diagonal (infinite) kinetic term is induced, a corresponding non-diagonal counter term should be added. Then after the diagonalization the originally assumed structure of the couplings in the superpotential will receive large quantum corrections. In other words, we have in spite of the non-renormalization theorem more parameters in the superpotential, when written in terms of the bare fields, than originally assumed. The undesired mixing among $\Phi_{i}$ 's and large quantum corrections can be avoided if an appropriate flavor symmetry is present.

We will see below that the non-renormalization theorem and the renormalization properties of the soft-supersymmetry-breaking (SSB) terms allow us to introduce in a consistent manner different flavor symmetries for different sectors of a softly broken supersymmetric theory to control the independent parameters of the theory.

To be more specific, we consider an $N=1$ supersymmetric gauge theory whose superpo- 
tential is given by

$$
W(\Phi)=W_{Y}(\Phi)+W_{\mu}(\Phi)
$$

with

$$
W_{Y}(\Phi)=\frac{1}{6} Y^{i j k} \Phi_{i} \Phi_{j} \Phi_{k} \text { and } W_{\mu}(\Phi)=\frac{1}{2} \mu^{i j} \Phi_{i} \Phi_{j}
$$

The SSB Lagrangian can be written as

$$
\begin{aligned}
L(\Phi, W)= & -\left(\int d^{2} \theta \eta\left(\frac{1}{6} h^{i j k} \Phi_{i} \Phi_{j} \Phi_{k}+\frac{1}{2} b^{i j} \Phi_{i} \Phi_{j}+\frac{1}{2} M_{g} W_{A}^{\alpha} W_{A \alpha}\right)+\text { h.c. }\right) \\
& -\int d^{4} \theta \tilde{\eta} \eta \overline{\Phi^{j}}\left(m^{2}\right)_{j}^{i}\left(e^{2 g V}\right)_{i}^{k} \Phi_{k}
\end{aligned}
$$

where $\eta=\theta^{2}, \tilde{\eta}=\tilde{\theta}^{2}$ are the external spurion superfields and $M_{g}$ is the gaugino mass. The $\beta$ functions of the $Y, \mu, h$ and $m^{2}$ are given by Refs. [33]-[41]

$$
\begin{aligned}
\beta_{Y}^{i j k} & =\gamma_{l}^{i} Y^{l j k}+\gamma^{j}{ }_{l} Y^{i l k}+\gamma_{l}^{k} Y^{i j l} \\
\beta_{\mu}^{i j} & =\gamma^{i} \mu^{l j}+\gamma_{l}{ }_{l} \mu^{i l} \\
\beta_{h}^{i j k} & =\gamma^{i} h^{l j k}+\gamma^{j} h^{i l k}+\gamma^{k}{ }_{l} h^{i j l}-2 \gamma_{1 l}^{i} Y^{l j k}-2 \gamma_{1}^{j} Y^{i l k}-2 \gamma_{1}^{k} Y^{i j l}, \\
\beta_{b}^{i j} & =\gamma^{i}{ }_{l} b^{l j}+\gamma^{j}{ }_{l} b^{i l}-2 \gamma_{1}^{i} \mu^{l j}-2 \gamma_{1}^{j} \mu^{i l} \\
\left(\beta_{m^{2}}\right)^{i}{ }_{j} & =\left[\Delta+X \frac{\partial}{\partial g}\right] \gamma^{i}{ }_{j}, \\
\mathcal{O} & =\left(M_{g} g^{2} \frac{\partial}{\partial g^{2}}-h^{l m n} \frac{\partial}{\partial Y^{l m n}}\right), \\
\Delta & =2 \mathcal{O O O}^{*}+2\left|M_{g}\right|^{2} g^{2} \frac{\partial}{\partial g^{2}}+\tilde{Y}_{l m n} \frac{\partial}{\partial Y_{l m n}}+\tilde{Y}^{l m n} \frac{\partial}{\partial Y^{l m n}}
\end{aligned}
$$

where $\left(\gamma_{1}\right)^{i}{ }_{j}=\mathcal{O} \gamma^{i}{ }_{j}, Y_{l m n}=\left(Y^{l m n}\right)^{*}$, and

$$
\begin{aligned}
\tilde{Y}^{i j k} & =\left(m^{2}\right)_{l}^{i} Y^{l j k}+\left(m^{2}\right)^{j}{ }_{l} Y^{i l k}+\left(m^{2}\right)^{k}{ }_{l} Y^{i j l}, \\
X & =\frac{-\left|M_{g}\right|^{2} C(G)+\sum_{l} m_{l}^{2} T\left(R_{l}\right)}{C(G)-8 \pi^{2} / g^{2}} .
\end{aligned}
$$

Here $X$ of (12) is the expression in the renormalization scheme of Novikov et al. [42], $T\left(R_{l}\right)$ is the Dynkin index of $R_{l}$, and $C_{2}(G)$ is the quadratic Casimir of the adjoint representation of the gauge group $G$. From Eqs. (41)-(12) we now derive the hierarchical structure of the renormalization properties of the theory, which is basically the Symanzik theorem applied to softly broken supersymmetric gauge theories:

1. The (infinite) renormalization of the supersymmetric parameters $Y^{i j k}, \mu^{i j}$ is not influenced by the SSB terms, in accord with the definition of the SSB terms.

2. The (infinite) renormalization of the tri-linear couplings $h^{i j k}$ does not depend on $\mu^{i j}$. It is also independent on $\left(m^{2}\right)_{j}^{i}$ and $b^{i j}$. 
3. The (infinite) renormalization of the soft scalar masses $\left(m^{2}\right)_{j}^{i}$ does not depend on $b^{i j}$ and $\mu^{i j}$, as one can see from Eqs. (8) $)$ (12).

4. The (infinite) renormalization of $b^{i j}$ does not depend $\left(m^{2}\right)_{j}^{i}$ and $h^{i j k}$, which is the consequence of (77).

Because of these renormalization properties we can consistently introduce different symmetries for different sectors.

To begin with we assume the existence of a flavor symmetry in the Yukawa sector which protects the mixing (of the wave function renormalization) among the matter superfields $\Phi_{i}{ }^{\prime}$ s. ${ }^{3}$ This implies that the anomalous dimensions $\gamma_{j}^{i}$ are diagonal, i.e.,

$$
\gamma_{j}^{i}=\delta_{j}^{i} \gamma_{j}
$$

Then Eqs. (4) -(8) become

$$
\begin{aligned}
\beta_{Y}^{i j k} & =Y^{i j k}\left(\gamma_{i}+\gamma_{j}+\gamma_{k}\right), \beta_{\mu}^{i j}=\mu^{i j}\left(\gamma_{i}+\gamma_{j}\right) \\
\beta_{h}^{i j k} & =\left(h^{i j k}-2 Y^{i j k} \mathcal{O}\right)\left(\gamma_{i}+\gamma_{j}+\gamma_{k}\right), \beta_{b}^{i j}=\left(b^{i j}-2 \mu^{i j} \mathcal{O}\right)\left(\gamma_{i}+\gamma_{j}\right), \\
\left(\beta_{m^{2}}\right)_{l} & =\left[\Delta+X \frac{\partial}{\partial g}\right] \gamma_{l}
\end{aligned}
$$

with $\tilde{Y}^{i j k}=Y^{i j k}\left(m_{i}^{2}+m_{j}^{2}+m_{k}^{2}\right)$. From these equations we observe:

(a) The $\mu$ sector can have a flavor symmetry which is different from the flavor symmetry of the Yukawa sector if both symmetries are compatible with respect to renormalization of $\mu_{i j}$.

(b) It is consistent to introduce into the tri-linear couplings the same flavor symmetry as that of the Yukawa couplings, even if it is violated in other sectors.

(c) The flavor symmetry which protects the mixing among $\Phi_{i}$ 's ensures that $\left(m^{2}\right)_{j}^{i}$ is diagonal. If the Yukawa couplings and tri-linear couplings have the flavor symmetry, the soft scalar mass terms, too, can have the flavor symmetry, even if the $\mu$ and $b$ terms do not respect the flavor symmetry.

(d) The $b$ terms associated with the $\mu$ terms should always exist (see (16)). But the $b$ sector has no influence on the infinite renormalization of the parameters in other sectors. So the violation of a symmetry in the $b$ sector is absolutely soft.

In the next section we reconsider the supersymmetric flavor model of [7, 21, 22] along the line of thought about a flavor symmetry in this section.

\footnotetext{
3 We also assume that the flavor symmetry is not gauged.
} 


\begin{tabular}{|c|c|c|c|c|c|c|c|c|c|c|c|}
\hline & $Q$ & $Q_{3}$ & $U^{c}, D^{c}$ & $U_{3}^{c}, D_{3}^{c}$ & $L$ & $L_{3}$ & $E^{c}, N^{c}$ & $E_{3}^{c}$ & $N_{3}^{c}$ & $H^{u}, H^{d}$ & $H_{3}^{u}, H_{3}^{d}$ \\
\hline$Q_{6}$ & $\mathbf{2}_{1}$ & $\mathbf{1}_{+, 2}$ & $\mathbf{2}_{2}$ & $\mathbf{1}_{-, 1}$ & $\mathbf{2}_{2}$ & $\mathbf{1}_{+, 0}$ & $\mathbf{2}_{2}$ & $\mathbf{1}_{+, 0}$ & $\mathbf{1}_{-, 3}$ & $\mathbf{2}_{2}$ & $\mathbf{1}_{-, 1}$ \\
\hline$R$ & - & - & - & - & - & - & - & - & - & + & + \\
\hline
\end{tabular}

TABLE I: The $Q_{6} \times R$ assignment of the chiral matter supermultiplets, where $R$ is the $R$ parity. The group theory notation is given in Ref. [7].

\section{THE MODEL}

The supersymmetric flavor model of [7, 21, 22] is based on a dicyclic dihedral group $Q_{6}$. If $\mathrm{CP}$ is spontaneously broken, the nine parameters of the model express six quark masses and four CKM parameters. In the leptonic sector there are only seven parameters to fix six lepton masses and six MNS parameters. As we announced in the introduction we would like to stress the one + two structure for each family; a $Q_{6}$ singlet and a $Q_{6}$ doublet for each family including the $S U(2)_{L}$ doublet Higgs fields.

\section{A. The Yukawa sector}

As in the original model of [7, 21, 22] we assume that the flavor symmetry of the Yukawa sector is based on $Q_{6}$. In Table I we write the $Q_{6}$ assignment of the quark, lepton and Higgs chiral supermultiplets, ${ }^{4}$ where $Q, Q_{3}, L, L_{3}$ and $H^{u}, H_{3}^{u}, H^{d}, H_{3}^{d}$ stand for $S U(2)_{L}$ doublet supermultiplets for the quarks, leptons and Higgs bosons, respectively. Similarly, $S U(2)_{L}$ singlet supermultiplets for quarks, charged leptons and neutrinos are denoted by $U^{c}, U_{3}^{c}, D^{c}, D_{3}^{c}, E^{c}, E_{3}^{c}$ and $N^{c}, N_{3}^{c}$. From Table I we see that the one+two structure of family is realized, and because of this structure the $Q_{6}$ flavor symmetry can ensure that no non-diagonal kinetic term can be induced. So (13) is satisfied.

We then write down the most general, renormalizable, $Q_{6} \times R$ invariant superpotential $W$ ( $R$ is the $R$ parity.):

$$
W_{Y}=W_{Q}+W_{L}
$$

where

$$
\begin{aligned}
W_{Q} & =\sum_{I, i, j, k=1,2,3}\left(Y_{i j}^{u I} Q_{i} U_{j}^{c} H_{I}^{u}+Y_{i j}^{d I} Q_{i} D_{j}^{c} H_{I}^{d}\right), \\
W_{L} & =\sum_{I, i, j, k=1,2,3}\left(Y_{i j}^{e I} L_{i} E_{j}^{c} H_{I}^{d}+Y_{i j}^{\nu I} L_{i} N_{j}^{c} H_{I}^{u}\right) .
\end{aligned}
$$

\footnotetext{
${ }^{4}$ The same model exists for $Q_{2 N}$ if $N$ is odd and a multiple of 3.
} 
The Yukawa matrices $Y^{\prime}$ s are given by

$$
\begin{aligned}
& \mathbf{Y}^{u 1(d 1)}=\left(\begin{array}{ccc}
0 & 0 & 0 \\
0 & 0 & Y_{b}^{u(d)} \\
0 & Y_{b^{\prime}}^{u(d)} & 0
\end{array}\right), \mathbf{Y}^{u 2(d 2)}=\left(\begin{array}{ccc}
0 & 0 & Y_{b}^{u(d)} \\
0 & 0 & 0 \\
-Y_{b^{\prime}}^{u(d)} & 0 & 0
\end{array}\right) \\
& \mathbf{Y}^{u 3(d 3)}=\left(\begin{array}{ccc}
0 & Y_{c}^{u(d)} & 0 \\
Y_{c}^{u(d)} & 0 & 0 \\
0 & 0 & Y_{a}^{u(d)}
\end{array}\right) \text {, } \\
& \mathbf{Y}^{e 1}=\left(\begin{array}{ccc}
-Y_{c}^{e} & 0 & Y_{b}^{e} \\
0 & Y_{c}^{e} & 0 \\
Y_{b^{\prime}}^{e} & 0 & 0
\end{array}\right), \mathbf{Y}^{e 2}=\left(\begin{array}{ccc}
0 & Y_{c}^{e} & 0 \\
Y_{c}^{e} & 0 & Y_{b}^{e} \\
0 & Y_{b^{\prime}}^{e} & 0
\end{array}\right), \mathbf{Y}^{e 3}=0 \\
& \mathbf{Y}^{\nu 1}=\left(\begin{array}{ccc}
-Y_{c}^{\nu} & 0 & 0 \\
0 & Y_{c}^{\nu} & 0 \\
Y_{b^{\prime}}^{\nu} & 0 & 0
\end{array}\right), \mathbf{Y}^{\nu 2}=\left(\begin{array}{ccc}
0 & Y_{c}^{\nu} & 0 \\
Y_{c}^{\nu} & & 0 \\
0 & Y_{b^{\prime}}^{\nu} & 0
\end{array}\right) \\
& \mathbf{Y}^{\nu 3}=\left(\begin{array}{ccc}
0 & 0 & 0 \\
0 & 0 & 0 \\
0 & 0 & Y_{a}^{\nu}
\end{array}\right)
\end{aligned}
$$

All the parameters appearing above are real, because we assume that $\mathrm{CP}$ is spontaneously broken. We will shortly come back to this issue.

\section{B. The $\mu$ sector}

The most general $Q_{6} \times R$ invariant renormalizable $\mu$ part of the superpotential is

$$
W_{\mu}^{\left(Q_{6}\right)}=\mu H_{I}^{u} H_{I}^{d}+\frac{m}{2} N_{I}^{c} N_{I}^{c}
$$

Note that no mass terms for $H_{3}^{u, d}$ and $N_{3}^{c}$ are allowed by $Q_{6}$ and that the superpotential $W_{\mu}^{\left(Q_{6}\right)}$ has an accidental $O(2)$ symmetry. For phenomenological reasons we however need mass terms for $H_{3}^{u, d}$ and $N_{3}^{c}$. Therefore, we assume that the flavor symmetry of the $\mu$ sector is $O(2)$ and that $H_{3}^{u, d}$ and $N_{3}^{c}$ are singlets of $O(2)$, and add

$$
W_{\mu}^{\left(\phi_{6}\right)}=\mu_{3} H_{3}^{u} H_{3}^{d}+\frac{m_{3}}{2} N_{3}^{c} N_{3}^{c}
$$

to (23). Then the total $\mu$ part of the superpotential is $W_{\mu}=W_{\mu}^{\left(Q_{6}\right)}+W_{\mu}^{\left(Q_{6}\right)}$. The $O(2) \times R$ symmetry of $W_{\mu}$ is compatible with $Q_{6} \times R$ of the Yukawa sector, because $Q_{6}$ ensures

$$
\gamma_{H_{1}^{u}}=\gamma_{H_{2}^{u}} \text { and } \gamma_{H_{1}^{d}}=\gamma_{H_{2}^{d}}
$$




\section{Soft-supersymmetry-breaking sector}

\section{The tri-linear couplings and soft scalar mass terms}

We require that the tri-linear couplings and soft scalar mass terms have the same flavor symmetry as that of the Yukawa sector, that is, $Q_{6} \times R$. Therefore, the tri-linear couplings and soft scalar mass matrices have the following form:

$$
\mathbf{h}_{i j}^{k}=A_{i j} \mathbf{Y}_{i j}^{k}, k=u 1, u 2, \ldots, \nu_{3},
$$

where $\mathbf{Y}_{i j}^{k}$ are given in (20)-(22), and

$$
\mathbf{m}^{2} \propto\left(\begin{array}{lll}
1 & 0 & 0 \\
0 & 1 & 0 \\
0 & 0 & f
\end{array}\right) .
$$

for all the bosonic scalar partners. This is very crucial to suppress FCNCs in the SSB sector as we will see later on.

\section{The b terms}

The $b$ sector should contain at least terms which correspond to the $\mu$ terms $W_{\mu}=W_{\mu}^{\left(Q_{6}\right)}+$ $W_{\mu}^{\left(Q_{6}\right)}$, where $W_{\mu}^{\left(Q_{6}\right)}$ and $W_{\mu}^{\left(Q_{6)}\right)}$ are given in (23) and (24), respectively, i.e.

$$
\mathcal{L}_{b}^{\left(O_{2}\right)}==b \hat{H}_{I}^{u} \hat{H}_{I}^{d}+b_{33} \hat{H}_{3}^{u} \hat{H}_{3}^{d}+b_{N} \hat{N}_{I}^{c} \hat{N}_{I}^{c}+b_{N_{3}} \hat{N}_{3}^{c} \hat{N}_{3}^{c}+\text { h.c. }
$$

(The hatted fields are bosonic components.) Because of the $O(2)$ symmetry in the $\mu$ and $b$ sectors and the $Q_{6}$ symmetry in the soft scalar mass terms, the Higgs scalar potential also respects the $O(2)$ symmetry, so that there is a Nambu-Goldstone boson corresponding to this symmetry because the $O(2)$ symmetry the gauge symmetry is spontaneously broken, together with $S U(2)_{L} \times U(1)_{Y}$. Moreover, we face the domain wall problem when the discrete flavor symmetries are spontaneously broken. To overcome these problems we add terms which explicitly break $O_{2}$ down to $Z_{2}$ :

$$
\begin{aligned}
\mathcal{L}_{b}^{\left(\phi_{2}\right)}== & b_{++} \hat{H}_{+}^{u} \hat{H}_{+}^{d}+b_{--} \hat{H}_{-}^{u} \hat{H}_{-}^{d}+b_{+3} \hat{H}_{+}^{u} \hat{H}_{3}^{d}+b_{3+} \hat{H}_{3}^{u} \hat{H}_{+}^{d} \\
& +b_{N_{+}} \hat{N}_{+}^{c} \hat{N}_{+}^{c}+b_{N_{-}} \hat{N}_{-}^{c} \hat{N}_{-}^{c}+\hat{N}_{3}^{c} \hat{N}_{+}^{c}+\text { h.c. },
\end{aligned}
$$

where

$$
H_{ \pm}^{u, d}=\frac{1}{\sqrt{2}}\left(H_{1}^{u, d} \pm H_{2}^{u, d}\right), N_{ \pm}^{c}=\frac{1}{\sqrt{2}}\left(N_{1}^{c} \pm N_{2}^{c}\right) .
$$

$\left(H_{+}^{u, d}, H_{3}^{u, d}, N_{+}^{c}\right.$ and $N_{3}^{c}$ are $Z_{2}$ even, while $H_{-}^{u, d}$ and $N_{-}^{c}$ are $Z_{2}$ odd.) This $Z_{2}$ is indeed broken by the the Yukawa and and tri-linear couplings, but is compatible with $Q_{6}$, i.e., $\gamma_{H_{1}^{u, d}}=\gamma_{H_{2}^{u, d}}$ 


\begin{tabular}{|c|c|c|c|c|}
\hline & $\mathbf{Y}, \mathbf{h}$ & $\mathbf{m}$ & $\mu$ sector & $b$ terms \\
\hline$Q_{6}$ & $\bigcirc$ & $\bigcirc$ & $\times$ & $\times$ \\
\hline$O_{2}$ & $\times$ & $\bigcirc$ & $\bigcirc$ & $\times$ \\
\hline$Z_{2}$ & $\times$ & $\bigcirc$ & $\bigcirc$ & $\bigcirc$ \\
\hline $\mathrm{CP}$ & $\bigcirc$ & $\bigcirc$ & $\bigcirc$ & $\times$ \\
\hline $\mathrm{R}$ & $\bigcirc$ & $\bigcirc$ & $\bigcirc$ & $\bigcirc$ \\
\hline
\end{tabular}

TABLE II: The symmetry of the different sectors. $\mathbf{Y}, \mathbf{h}$ and $\mathbf{m}$ stand for the Yukawa, tri-linear and soft scalar mass sector, respectively. $Q_{6}$ ensures that all the anomalous dimensions $\gamma^{\prime}$ 's are diagonal, and that the two components of a $Q_{6}$ doublet have a same anomalous dimension. Therefore, $Q_{6}$ in the Yukawa and tri-linear sectors and $\mathrm{O}_{2}$ in the $\mu$ sector are compatible with each other. $\mathrm{O}_{2}$ in the soft scalar mass sector is accidental. $Z_{2}$ is a subgroup of $O_{2}$, which implies the compatibility of $O_{2}$ and $Z_{2}$. CP is explicitly broken only by the $b$ terms, which is (super) soft because the propagation of its violation to the other sectors is calculable and small. So, all the symmetries are compatible with each other.

We allow the $b$ parameters to be complex, because $\mathrm{CP}$ can not be broken if all the $b$ parameters are real as we will find in the next subsection. So CP is explicitly, but only softly broken in this sector. In Table [I] we give the symmetry of the each sector.

\section{THE HIGGS SECTOR}

\section{A. The Higgs potential}

Given the $O(2) \times R$ invariant superpotential $W_{\mu}$ in the $\mu$ sector (23) and (24) along with the $Q_{6} \times R$ invariant soft scalar masses (27) and the $Z_{2} \times R$ invariant $b$ terms (28) and (29), we can now write down the scalar potential. For simplicity we assume that only the neutral scalar components (denoted by a superscript 0) of the Higgs supermultiplets acquire VEVs:

$$
\begin{aligned}
V & =m_{H_{+}^{u}}^{2}\left(\left|\hat{H}_{+}^{0 u}\right|^{2}+\left|\hat{H}_{-}^{0 u}\right|^{2}\right)+m_{H_{+}^{d}}^{2}\left(\left|\hat{H}_{+}^{0 d}\right|^{2}+\left|\hat{H}_{-}^{0 d}\right|^{2}\right)+m_{H_{3}^{u}}^{2}\left|\hat{H}_{3}^{0 u}\right|^{2}+m_{H_{3}^{d}}^{2}\left|\hat{H}_{3}^{0 d}\right|^{2} \\
& +\frac{1}{8}\left(\frac{3}{5} g_{1}^{2}+g_{2}^{2}\right)\left(\left|\hat{H}_{+}^{0 u}\right|^{2}+\left|\hat{H}_{-}^{0 u}\right|^{2}+\left|\hat{H}_{3}^{0 u}\right|^{2}-\left|\hat{H}_{+}^{0 d}\right|^{2}-\left|\hat{H}_{-}^{0 d}\right|^{2}-\left|\hat{H}_{3}^{0 d}\right|^{2}\right)^{2} \\
& +\left[b_{++}^{\prime} \hat{H}_{+}^{0 u} \hat{H}_{+}^{0 d}+b_{--}^{\prime} \hat{H}_{-}^{0 u} \hat{H}_{-}^{0 d}+b_{+3} \hat{H}_{+}^{0 u} \hat{H}_{3}^{0 d}+b_{3+} \hat{H}_{3}^{0 u} \hat{H}_{+}^{0 d}+b_{33} \hat{H}_{3}^{0 u} \hat{H}_{3}^{0 d}+h . c .\right]
\end{aligned}
$$

where $b_{++(-)}^{\prime}=b+b_{++(--)}, g_{1,2}$ are the gauge coupling constants for the $U(1)_{Y}$ and $S U(2)_{L}$ gauge groups, and $H_{ \pm}$'s are defined in (30). Note that the scalar potential (31) has the same $Z_{2}$ symmetry as that of the $b$ sector. ( $H_{+}$'s and $H_{3}$ 's are $Z_{2}$ even, and $H_{-}$'s are $Z_{2}$ odd.) Therefore,

$$
<\hat{H}_{-}^{0 u, d}>=0,<\hat{H}_{+}^{0 u, d}>=\frac{v_{+}^{u, d}}{\sqrt{2}} \exp i \theta_{+}^{u, d},<\hat{H}_{3}^{0 u, d}>=\frac{v_{3}^{u, d}}{\sqrt{2}} \exp i \theta_{3}^{u, d}
$$


can become a local minimum, where we assume that $v_{+}^{u, d}$ and $v_{3}^{u, d}$ are real. We recall that the $Z_{2}$ is an accidental symmetry expect for the $b$ sector. ${ }^{5}$ Therefore, the VEV structure (32) is stable against (infinite) renormalization.

We investigate whether the potential energy at the VEV (32) can become negative so that $S U(2)_{L} \times U(1)_{Y}$ is spontaneously broken. To this end we consider the quadratic part of the scalar potential

$$
V^{(2)}=\mathcal{H}^{I} \mathcal{M}_{I J} \mathcal{H}^{J}
$$

where

$$
\mathcal{M}=\left(\begin{array}{cccccccc}
m_{H_{+}^{u}}^{2} & 0 & \Re\left(b_{++}^{\prime}\right) & -\Im\left(b_{++}^{\prime}\right) & 0 & 0 & \Re\left(b_{+3}\right) & -\Im\left(b_{+3}\right) \\
0 & m_{H_{+}^{u}}^{2} & -\Im\left(b_{++}^{\prime}\right) & -\Re\left(b_{++}^{\prime}\right) & 0 & 0 & -\Im\left(b_{+3}\right) & -\Re\left(b_{+3}\right) \\
\Re\left(b_{++}^{\prime}\right) & -\Im\left(b_{++}^{\prime}\right) & m_{H_{+}^{d}}^{2} & 0 & \Re\left(b_{3+}\right) & -\Im\left(b_{3+}\right) & 0 & 0 \\
-\Im\left(b_{++}^{\prime}\right) & -\Re\left(b_{++}^{\prime}\right) & 0 & m_{H_{+}^{d}}^{2} & -\Im\left(b_{3+}\right) & -\Re\left(b_{3+}\right) & 0 & 0 \\
0 & 0 & \Re\left(b_{3+}\right) & -\Im\left(b_{3+}\right) & m_{H_{3}^{u}}^{2} & 0 & \Re\left(b_{33}\right) & -\Im\left(b_{33}\right) \\
0 & 0 & -\Im\left(b_{3+}^{\prime}\right) & -\Re\left(b_{3+}\right) & 0 & m_{H_{3}^{u}}^{2} & -\Im\left(b_{33}\right) & -\Re\left(b_{33}\right) \\
\Re\left(b_{+3}\right) & -\Im\left(b_{+3}\right) & 0 & 0 & \Re\left(b_{33}\right) & -\Im\left(b_{33}\right) & m_{H_{3}^{d}}^{2} & 0 \\
-\Im\left(b_{+3}\right) & -\Re\left(b_{+3}\right) & 0 & 0 & -\Im\left(b_{33}\right) & -\Re\left(b_{33}\right) & 0 & m_{H_{3}^{d}}^{2}
\end{array}\right),
$$

and

$$
\mathcal{H}=\left(\Re\left(\hat{H}_{+}^{0 u}\right), \Im\left(\hat{H}_{+}^{0 u}\right), \Re\left(\hat{H}_{+}^{0 d}\right), \Im\left(\hat{H}_{+}^{0 d}\right), \Re\left(\hat{H}_{3}^{0 u}\right), \Im\left(\hat{H}_{3}^{0 u}\right), \Re\left(\hat{H}_{3}^{0 d}\right), \Im\left(\hat{H}_{3}^{0 d}\right)\right)
$$

We find that all the eigenvalues of $\mathcal{M}$ are doubly generate, and that two orthogonal eigenvectors of the same eigenvalue can be always written in the form

$$
\vec{u}_{A}=\left(u_{1}, u_{2}, u_{3}, u_{4}, u_{5}, u_{6}, u_{7}, u_{8}\right) \text { and } \vec{u}_{B}=\left(u_{2},-u_{1},-u_{4}, u_{3}, u_{6},-u_{5},-u_{8}, u_{7}\right) .(36
$$

This is due to the $U(1)_{Y}$ gauge invariance: All the directions defined by a linear combination of $\vec{u}_{A}$ and $\vec{u}_{B}$ are physically equivalent. If all the imaginary parts of $b$ 's vanish, then we find $u_{2}=u_{4}=u_{6}=u_{8}=0$, which means that CP can not be spontaneously broken, because the imaginary parts $\Im\left(\mathcal{H}_{I}\right)$ along the direction defined by $\left(u_{1}, 0, u_{3}, 0, u_{5}, 0, u_{7}, 0\right)$ stay at zero. So at least one of $b$ 's should be complex so that CP is spontaneously broken. ${ }^{6}$ The product of the four independent eigenvalues is $\operatorname{det} \mathcal{M}$. Therefore, if $\operatorname{det} \mathcal{M}$ is negative, one

\footnotetext{
${ }^{5}$ It is accidental in the part of (31) coming from the D-terms (the second line). The $Q_{6}$ invariant soft scalar mass terms respect automatically this $Z_{2}$, although it is not contained in $Q_{6}$. This $Z_{2}$ is a part of the $O(2)$ symmetry of the $\mu$ sector, which is only softly broken down to the $Z_{2}$ in the $b$ sector.

6 Spontaneous CP violation in supersymmetric models and two Higgs doublet models have been discussed in Refs. [43]- 49], Ref. [50] and references therein.
} 
or three independent eigenvalues are negative. If $\operatorname{det} \mathcal{M}$ is positive, there may be zero, two or four negative eigenvalues. In this case one should compute the eigenvalues explicitly. A local minimum lies along the direction of a negative eigenvalue. Further, the potential (31) along the D-term flat direction should not be unbounded below. This condition requires

$$
\begin{aligned}
& m_{H_{+}^{u}}^{2}+m_{H_{+}^{d}}^{2}-2\left|b_{++}^{\prime}\right|>0, m_{H_{+}^{u}}^{2}+m_{H_{+}^{d}}^{2}-2\left|b_{--}^{\prime}\right|>0, m_{H_{+}^{u}}^{2}+m_{H_{3}^{d}}^{2}-2\left|b_{+3}\right|>0, \\
& m_{H_{3}^{u}}^{2}+m_{H_{+}^{d}}^{2}-2\left|b_{3+}\right|>0, m_{H_{3}^{u}}^{2}+m_{H_{3}^{d}}^{2}-2\left|b_{33}\right|>0 .
\end{aligned}
$$

We have to make the flavor changing neutral Higgs bosons sufficiently heavy to suppress FCNCs. (This will be discussed in Sec. V.) So we need a certain fine tuning among the SSB parameters, because the size of the VEVs is bounded from above. To achieve this situation, we have to so fine tune the parameters that one negative eigenvalue at the origin of the potential becomes very small. 7 Then the potential energy falls only slowly when moving from the origin, and the quartic terms in the potential (31) coming from the D-terms start to dominate, so that the energy scale of the VEVs at the bottom of the potential can be much smaller then the energy scale of the SSB parameters. Here is such an example:

$$
\begin{aligned}
\Im\left(b_{++}\right) / \Re\left(b_{++}^{\prime}\right) & =0.747, \Re\left(b_{33}\right) / \Re\left(b_{++}^{\prime}\right)=0.852, \Im\left(b_{33}\right) / \Re\left(b_{++}^{\prime}\right)=1.399, \\
\Re\left(b_{+3}\right) / \Re\left(b_{++}^{\prime}\right) & =0.667, \Im\left(b_{+3}\right) / \Re\left(b_{++}^{\prime}\right)=0.31, \\
\Re\left(b_{3+}\right) / \Re\left(b_{++}^{\prime}\right) & =1.3, \Im\left(b_{3+}\right) / \Re\left(b_{++}^{\prime}\right)=0.42, \\
m_{H_{+}^{u}}^{2} / \Re\left(b_{++}^{\prime}\right) & =3.13, m_{H_{+}^{d}}^{2} / \Re\left(b_{++}^{\prime}\right)=2.69, \\
m_{H_{3}^{u}}^{2} / \Re\left(b_{++}^{\prime}\right) & =1.39, m_{H_{3}^{d}}^{2} / \Re\left(b_{++}^{\prime}\right)=5.93 .
\end{aligned}
$$

The four independent eigenvalues are $-5.4 \times 10^{-5}, 2.27,4.16,6.70$ in the unit of $b_{++}^{\prime}$, and two eigenvectors for the smallest eigenvalue correspond to

$$
\begin{aligned}
& u_{1}=-0.1070, u_{2}=0.2232, u_{3}=0.4091, u_{4}=0.3081, \\
& u_{5}=-0.4216, u_{6}=0.6636, u_{7}=0.2408, u_{8}=0.0154,
\end{aligned}
$$

where $u$ 's are defined in (36). Along the direction defined by (39) the potential energy falls very slowly when moving from the origin. So the $S U(2)_{L} \times U(1)_{Y}$ invariant point is a saddle point, and we find that the size of $\sqrt{b_{++}^{\prime}}$ may be estimated as

$$
\sqrt{b_{++}^{\prime}} \simeq\left(\frac{0.13\left(g_{2}^{2}+3 g_{1}^{2} / 5\right) / 8}{5.4 \times 10^{-5}}\right)^{1 / 2} \times(246 \mathrm{GeV}) \simeq 3.2 \mathrm{TeV} .
$$

$\mathrm{CP}$ is also spontaneously broken, because it is not possible to obtain a vector of the form $(\bullet, 0, \bullet, 0, \bullet, 0, \bullet, 0)$ through a linear combination of $\vec{u}_{A}$ and $\vec{u}_{B}$ for (39). Therefore, the angle $\theta_{q}$ that enters in the calculation of the CKM (given in (67)) is non-zero for (39). We find:

$$
\begin{aligned}
\theta_{q} & =\theta_{+}^{u}-\theta_{+}^{d}-\theta_{3}^{u}+\theta_{3}^{d} \\
& =\arctan \left(u_{2} / u_{1}\right)-\arctan \left(u_{4} / u_{3}\right)-\arctan \left(u_{6} / u_{5}\right)+\arctan \left(u_{8} / u_{7}\right) \simeq-0.701
\end{aligned}
$$

\footnotetext{
7 By one eigenvalue we mean one of four eigenvalues. All the eigenvalues are doubly degenerate.
} 
which is the size of $\theta_{q}$ we need to produce the correct CKM parameters as we will see in Sec. V.

\section{B. The heavy neutral Higgs fields}

Now redefine the Higgs fields as follows: First we define the tilde fields

$$
\tilde{H}_{+}^{0 u, 0 d}=\hat{H}_{+}^{0 u, 0 d} \exp -i \theta_{+}^{u, d}, \tilde{H}_{3}^{0 u, 0 d}=\hat{H}_{3}^{0 u, 0 d} \exp -i \theta_{3}^{u, d},
$$

and then

$$
\phi_{L}^{u}=\cos \gamma^{u} \tilde{H}_{3}^{0 u}+\sin \gamma^{u} \tilde{H}_{+}^{0 u}, \phi_{H}^{u}=-\sin \gamma^{u} \tilde{H}_{3}^{0 u}+\cos \gamma^{u} \tilde{H}_{+}^{0 u}
$$

where

$$
\cos \gamma^{u}=\frac{v_{3}^{u}}{\sqrt{\left(v_{3}^{u}\right)^{2}+\left(v_{+}^{u}\right)^{2}}}, \quad \sin \gamma^{u}=\frac{v_{+}^{u}}{\sqrt{\left(v_{3}^{u}\right)^{2}+\left(v_{+}^{u}\right)^{2}}}
$$

and similarly for the down sector. As we see from (44), only $\phi_{L}^{u}$ and $\phi_{L}^{d}$ have a nonvanishing $\mathrm{VEV}$, which we denote by

$$
<\phi_{L}^{u, d}>=\frac{\sqrt{\left(v_{3}^{u, d}\right)^{2}+\left(v_{+}^{u, d}\right)^{2}}}{\sqrt{2}}=\frac{v_{u, d}}{\sqrt{2}} .
$$

The neutral light and heavy scalars of the MSSM are given by

$$
\begin{aligned}
\frac{1}{\sqrt{2}}(v+h) & =\operatorname{Re}\left(\phi_{L}^{d *}\right) \cos \beta+\operatorname{Re}\left(\phi_{L}^{u}\right) \sin \beta, \\
\frac{1}{\sqrt{2}}(H+i A) & =-\left(\phi_{L}^{d *}\right) \sin \beta+\left(\phi_{L}^{u}\right) \cos \beta,
\end{aligned}
$$

where as in the MSSM

$$
v=\sqrt{v_{u}^{2}+v_{d}^{2}}, \tan \beta=\frac{v_{u}}{v_{d}}
$$

As in the case of the MSSM, the couplings of $\phi_{L}^{u, d}$ are flavor-diagonal, and so we do not have to consider them below when discussing FCNCs. Therefore, only the heavy fields $\hat{H}_{-}^{0 u, 0 d}=\phi_{-}^{u, d}$ and $\phi_{H}^{u, d}$ can have flavor-changing couplings. Their mass matrix can be written as

$$
\left(\begin{array}{cccc}
m_{\phi_{I}^{u}}^{2} & 0 & 0 & b_{I}^{*} \\
0 & m_{\phi_{I}^{u}}^{2} & b_{I} & 0 \\
0 & b_{I}^{*} & m_{\phi_{I}^{d}}^{2} & 0 \\
b_{I} & 0 & 0 & m_{\phi_{I}^{d}}^{2}
\end{array}\right)
$$


in the $\left(\phi_{I}^{u}, \phi_{I}^{u *}, \phi_{I}^{d}, \phi_{I}^{d *}\right)$ basis, where $I=-, H$,

$$
\begin{aligned}
m_{\phi_{-}^{u, d}}^{2} & =m_{H_{-}^{u, d}}^{2}, b_{-}=b_{--}^{\prime}, m_{\phi_{H}^{u, d}}^{2}=m_{H_{+}^{u, d}}^{2} \cos ^{2} \gamma^{u, d}+m_{H_{3}^{u, d}}^{2} \sin ^{2} \gamma^{u, d} \\
b_{H} & =b_{++}^{\prime} e^{-i\left(\theta_{+}^{u}+\theta_{+}^{d}\right)} \cos \gamma^{u} \cos \gamma^{d}-b_{+3} \cos \gamma^{u} \sin \gamma^{d} e^{-i\left(\theta_{+}^{u}+\theta_{3}^{d}\right)} \\
& -b_{3+} \sin \gamma^{u} \cos \gamma^{d} e^{-i\left(\theta_{3}^{u}+\theta_{+}^{d}\right)}+b_{33} \sin \gamma^{u} \sin \gamma^{d} e^{-i\left(\theta_{3}^{u}+\theta_{3}^{d}\right)}
\end{aligned}
$$

and the mass parameters on the rhs are given in (31) and $\gamma^{u, d}$ are defined in (44). The inverse of the matrix (49) is given by

$$
\frac{1}{\left(M_{I 1}\right)^{2}\left(M_{I 2}\right)^{2}}\left(\begin{array}{cccc}
m_{\phi_{I}^{d}}^{2} & 0 & 0 & -b_{I}^{*} \\
0 & m_{\phi_{I}^{d}}^{2} & -b_{I} & 0 \\
0 & -b_{I}^{*} & m_{\phi_{I}^{u}}^{2} & 0 \\
-b_{I} & 0 & 0 & m_{\phi_{I}^{u}}^{2}
\end{array}\right) \quad, \quad(I=-, H)
$$

where $M_{1,2}$ are approximate pole masses and given by

$$
\left(M_{I 1(2)}\right)^{2}=\frac{1}{2}\left(m_{\phi_{I}^{u}}^{2}+m_{\phi_{I}^{d}}^{2}\right)\left(1+(-)\left[\frac{4\left|b_{I}\right|^{2}+\left(m_{\phi_{I}^{u}}^{2}-m_{\phi_{I}^{d}}^{2}\right)^{2}}{\left(m_{\phi_{I}^{u}}^{2}+m_{\phi_{I}^{d}}^{2}\right)^{2}}\right]^{1 / 2}\right),
$$

and we find

$$
\left(M_{I 1}\right)^{2}\left(M_{I 2}\right)^{2}=-\left|b_{I}\right|^{2}+m_{\phi_{I}^{u}}^{2} m_{\phi_{I}^{d}}^{2}
$$

(51) is the inverse propagator at the zero momentum. We will be using it later on. For the parameter values in the example (39) we find

$$
\begin{aligned}
\tan \gamma^{u} & =0.315, \tan \gamma^{d}=2.122, \tan \beta=-1.456 \\
M_{H 1} & =2.31 \sqrt{b_{++}^{\prime}} \simeq 7.3 \mathrm{TeV}, M_{H 2}=1.72 \sqrt{b_{++}^{\prime}} \simeq 5.5 \mathrm{TeV},
\end{aligned}
$$

where we have used (40). So, what we have numerically shown in A and B in this section is that it is possible to fine tune the SSB parameters so as to make the heavy Higgs bosons

much heavier than $M_{Z}$ (see (54) ) and at the same time to obtain a desired size of spontaneous $\mathrm{CP}$ violation (see (41)).

\section{FCNCS}

\section{A. The physical quarks and leptons}

From the Yukawa interactions (18) and (19) along with the form of the VEVs (32) we obtain the fermion mass matrices. 


\section{Quark sector}

The quark mass matrices are given by

$$
\begin{aligned}
\mathbf{m}^{u} & =\frac{1}{2}\left(\begin{array}{ccc}
0 & \sqrt{2} Y_{c}^{u} v_{3}^{u} e^{-i \theta_{3}^{u}} & Y_{b}^{u} v_{+}^{u} e^{-i \theta_{+}^{u}} \\
\sqrt{2} Y_{c}^{u} v_{3}^{u} e^{-i \theta_{3}^{u}} & 0 & Y_{b}^{u} v_{+}^{u} e^{-i \theta_{+}^{u}} \\
-Y_{b^{\prime}}^{u} v_{+}^{u} e^{-i \theta_{+}^{u}} & Y_{b^{\prime}}^{u} v_{+}^{u} e^{-i \theta_{+}^{u}} & \sqrt{2} Y_{a}^{u} v_{3}^{u} e^{-i \theta_{3}^{u}}
\end{array}\right), \\
\mathbf{m}^{d} & =\frac{1}{2}\left(\begin{array}{ccc}
0 & \sqrt{2} Y_{c}^{d} v_{3}^{d} e^{-i \theta_{3}^{d}} & Y_{b}^{d} v_{+}^{d} e^{-i \theta_{+}^{d}} \\
\sqrt{2} Y_{c}^{d} v_{3}^{d} e^{-i \theta_{3}^{d}} & 0 & Y_{b}^{d} v_{+}^{d} e^{-i \theta_{+}^{d}} \\
-Y_{b^{\prime}}^{d} v_{+}^{d} e^{-i \theta_{+}^{d}} & Y_{b^{\prime}}^{d} v_{+}^{d} e^{-i \theta_{+}^{d}} & \sqrt{2} Y_{a}^{d} v_{3}^{d} e^{-i \theta_{3}^{d}}
\end{array}\right) .
\end{aligned}
$$

Then using the phase matrices defined below

$$
\begin{aligned}
R_{L} & =\frac{1}{\sqrt{2}}\left(\begin{array}{ccc}
1 & 1 & 0 \\
-1 & 1 & 0 \\
0 & 0 & \sqrt{2}
\end{array}\right), R_{R}=\frac{1}{\sqrt{2}}\left(\begin{array}{ccc}
-1 & -1 & 0 \\
-1 & 1 & 0 \\
0 & 0 & \sqrt{2}
\end{array}\right) \\
P_{L}^{u} & =\left(\begin{array}{ccc}
1 & 0 & 0 \\
0 & \exp \left(i 2 \Delta \theta^{u}\right) & 0 \\
0 & 0 & \exp \left(i \Delta \theta^{u}\right)
\end{array}\right), \\
P_{R}^{u} & =\left(\begin{array}{ccc}
\exp \left(i 2 \Delta \theta^{u}\right) & 0 & 0 \\
0 & 1 & 0 \\
0 & 0 & \exp \left(i \Delta \theta^{u}\right)
\end{array}\right) \exp \left(i \theta_{3}^{u}\right), \\
\Delta \theta^{u} & =\theta_{3}^{u}-\theta_{+}^{u},
\end{aligned}
$$

and similarly for the down sector, we can bring $\mathbf{m}^{u}$ into a real form

$$
\hat{\mathbf{m}}^{u}=P_{L}^{u \dagger} R_{L}^{T} \mathbf{m}^{u} R_{R} P_{R}^{u}=m_{t}\left(\begin{array}{ccc}
0 & q_{u} / y_{u} & 0 \\
-q_{u} / y_{u} & 0 & b_{u} \\
0 & b_{u}^{\prime} & y_{u}^{2}
\end{array}\right) .
$$

The mass matrix $\hat{\mathbf{m}}^{u}$ can then be diagonalized as

$$
O_{L}^{u T} \hat{\mathbf{m}}^{u} O_{R}^{u}=\left(\begin{array}{ccc}
m_{u} & 0 & \\
0 & m_{c} & 0 \\
0 & 0 & m_{t}
\end{array}\right)
$$

and similarly for $\mathbf{m}^{d}$, where $O_{L, R}^{u, d}$ are orthogonal matrices. So the mass eigenstates $u_{i L}^{\prime}=$ $\left(u_{L}^{\prime}, c_{L}^{\prime}, t_{L}^{\prime}\right)$ etc. can be obtained from

$$
u_{L}=U_{L}^{u} u_{L}^{\prime}, u_{R}=U_{R}^{u} u_{R}^{\prime}, d_{L}=U_{L}^{d} d_{L}^{\prime}, d_{R}=U_{R}^{d} d_{R}^{\prime}
$$

where

$$
U_{u L(R)}=R_{L(R)} P_{L(R)}^{u} O_{L(R)}^{u}
$$


Therefore, the CKM matrix $V_{\mathrm{CKM}}$ is given by

$$
V_{\mathrm{CKM}}=O_{L}^{u T} R_{L}^{T} R_{R} P_{L}^{u \dagger} O_{L}^{d}=O_{L}^{u T} P_{q} O_{L}^{d}
$$

where

$$
P_{q}=P_{L}^{u \dagger} P_{L}^{d}=\left(\begin{array}{ccc}
1 & 0 & 0 \\
0 & \exp \left(i 2 \Delta \theta_{q}\right) & 0 \\
0 & 0 & \exp \left(i \Delta \theta_{q}\right)
\end{array}\right)
$$

For the set of the parameters

$$
\begin{aligned}
& \theta_{q}=\theta_{3}^{d}-\theta_{+}^{d}-\theta_{3}^{u}+\theta_{+}^{u}=-0.7, q_{u}=0.0001799, b_{u}=0.05979, b_{u}^{\prime}=0.07054 \\
& y_{u}=0.99786, q_{d}=0.003784, b_{d}=0.03268, b_{d}^{\prime}=0.4620, y_{d}=-0.9415
\end{aligned}
$$

we obtain

$$
\begin{aligned}
m_{u} / m_{t} & =0.766 \times 10^{-5}, m_{c} / m_{t}=4.23 \times 10^{-3}, m_{d} / m_{b}=0.895 \times 10^{-3}, m_{s} / m_{b}=1.60 \times 10^{-2}, \\
\left|V_{\mathrm{CKM}}\right| & =\left(\begin{array}{ccc}
0.9740 & 0.2266 & 0.00362 \\
0.2265 & 0.9731 & 0.0417 \\
0.00849 & 0.0410 & 0.9991
\end{array}\right),\left|V_{t d} / V_{t s}\right|=0.207 \\
\sin 2 \beta\left(\phi_{1}\right) & =0.690, \gamma\left(\phi_{3}\right)=63.4^{o} .
\end{aligned}
$$

The experimental values to be compared are [51] (see also [52]):

$$
\begin{aligned}
& \left|V_{\mathrm{CKM}}^{\exp }\right|=\left(\begin{array}{rrr}
0.97383_{-0.00023}^{+0.00024} & 0.2272_{-0.0010}^{+0.0010} & 0.00396 \underset{-0.00009}{+0.00009} \\
0.2271_{-0.0010}^{+0.0010} & 0.97296_{-0.00024}^{+0.00024} & 0.04221_{-0.00080}^{+0.00010} \\
0.00814_{-0.00064}^{+0.00032} & 0.04161_{-0.00078}^{+0.00012} & 0.999100_{-0.000004}^{+0.000034}
\end{array}\right), \\
& \sin 2 \beta\left(\phi_{1}\right)=0.687 \pm 0.032,,\left|V_{t d} / V_{t s}\right|=0.208_{-0.006}^{+0.008} .
\end{aligned}
$$

The quark masses at $M_{Z}$ are given by [53]

$$
\begin{aligned}
m_{u} / m_{d} & =0.541 \pm 0.086(0.51), m_{s} / m_{d}=18.9 \pm 1.6(17.9) \\
m_{c} & =0.73 \pm 0.17(0.74) \mathrm{GeV}, m_{s}=0.058 \pm 0.015(0.046) \mathrm{GeV} \\
m_{t} & =175 \pm 6 \mathrm{GeV}, m_{b}=2.91 \pm 0.07 \mathrm{GeV}
\end{aligned}
$$

where the values in the parentheses are the theoretical values obtained from (68) for $m_{t}=174$ $\mathrm{GeV}$ and $m_{b}=2.9 \mathrm{GeV}$. So, we see that the model can well reproduce the experimentally measured parameters. 
The orthogonal matrices (62) are found to be

$$
\begin{aligned}
& O_{u L} \simeq\left(\begin{array}{ccc}
0.9991 & -0.04252 & 1.269 \times 10^{-5} \\
0.04244 & 0.9973 & 0.05964 \\
-2.548 \times 10^{-3} & -0.05958 & 0.9982
\end{array}\right) \\
& O_{u R} \simeq\left(\begin{array}{ccc}
-0.9991 & -0.04255 & -1.075 \times 10^{-5} \\
0.04244 & -0.9966 & 0.07042 \\
-3.007 \times 10^{-3} & 0.07035 & 0.9975
\end{array}\right) \\
& O_{d L} \simeq\left(\begin{array}{ccc}
0.9764 & 0.2160 & -1.856 \times 10^{-3} \\
-0.2159 & 0.9760 & 0.02899 \\
8.074 \times 10^{-3} & -0.02790 & 0.9996
\end{array}\right) \\
& O_{d R} \simeq\left(\begin{array}{ccc}
-0.9695 & 0.2452 & 1.165 \times 10^{-4} \\
-0.2174 & -0.8599 & 0.4618 \\
0.1133 & 0.4477 & 0.8870
\end{array}\right)
\end{aligned}
$$

\section{Lepton sector}

The charged lepton mass matrix becomes

$$
\mathbf{m}_{e}=\left(\begin{array}{ccc}
-m_{2} & m_{2} & m_{5} \\
m_{2} & m_{2} & m_{5} \\
m_{4} & m_{4} & 0
\end{array}\right) \exp \left(-i \theta_{+}^{d}\right)
$$

where

$$
m_{2}=\frac{1}{2} Y_{c}^{e} v_{+}^{d}, m_{4}=\frac{1}{2} Y_{b^{\prime}}^{e} v_{+}^{d}, m_{5}=\frac{1}{2} Y_{b}^{e} v_{+}^{d}
$$

The phase $\exp \left(-i \theta_{+}^{d}\right)$ can be rotated away, and all the mass parameters appearing in (76) are real. Diagonalization of the mass matrices is straightforward.

We would like to mention that the model has many predictions in this sector, because there are only four parameters to describe three light neutrino masses, three angles and three CP violating phases of $V_{M N S}$. Since the details of the predictions are presented in Refs. [23, 24, 54], we do not repeat them here again. ${ }^{8}$ Furthermore, the FCNC processes in the lepton sector have been very recently analyzed in details in Ref. [25], concluding that the model predictions of tree-level FCNC processes are at least five orders of magnitude smaller than the experimental upper bounds (The mass of the heavy neutral Higgs fields are

\footnotetext{
8 See also [55] for the predictions of the model on $\mathrm{R}$ parity violating processes. The leptonic sector of the present model is basically the same as the model of [23, 24], except for the spontaneous breaking of CP, which reduces one more independent phase in the leptonic sector.
} 
assumed to be $120 \mathrm{GeV}$.) For instance, the branching fraction for $\mu \rightarrow e \gamma$ is seven orders of magnitude smaller than the expected experimental sensitivity [25]. Therefore, we shall not consider FCNCs in the leptonic sector in the following discussions.

\section{B. CP violations and FCNCs in the SSB sector}

If three generations of a family have the one+two structure, then the soft scalar mass matrices for the sfermions have a diagonal form (27):

$$
\tilde{\mathbf{m}}_{a L L(R R)}^{2}=m_{\tilde{a}}^{2}\left(\begin{array}{ccc}
a_{L(R)}^{a} & 0 & 0 \\
0 & a_{L(R)}^{a} & 0 \\
0 & 0 & b_{L(R)}^{a}
\end{array}\right) \quad(a=u, d, e)
$$

where $m_{\tilde{a}}$ denote the average of the squark and slepton masses, respectively, and $\left(a_{L(R)}, b_{L(R)}\right)$ are dimensionless free real parameters of $O(1)$. Because of the $Q_{6}$ flavor symmetry in the trilinear interactions, all the soft left-right mass matrices assume the form

$$
\left(\tilde{\mathbf{m}}_{a L R}^{2}\right)_{i j}=A_{i j}^{a}\left(\mathbf{m}^{a}\right)_{i j} \quad(a=u, d, e)
$$

where $A_{i j}^{a}$ are free parameters of dimension one (see (26) $)$. They are also real, because we impose CP invariance in the tri-linear couplings.

The quantities [56, 57]

$$
\Delta_{L L(R R)}^{a}=U_{a L}^{\dagger} \tilde{\mathbf{m}}_{a L L(R R)}^{2} U_{a L(R)} \text { and } \Delta_{L R}^{a}=U_{a L}^{\dagger} \tilde{\mathbf{m}}_{a L R}^{2} U_{a R}
$$

in the super CKM basis are used widely to parameterize FCNCs and CP violations coming from the SSB sector, where the unitary matrices $U$ 's are given in (72)-(75).

\section{CP violations}

The imaginary parts of $\Delta$ 's (80) contribute to CP violating processes in the SSB sector. Recall that the soft scalar mass matrices $\mathbf{m}_{a L L, R R}^{2}$ are real, because they are diagonal, and that the phases of $\mathbf{m}_{a L R}^{2}$ come from the complex VEVs (32), because CP is only spontaneously broken in this sector. The unitary matrices U's are complex, and so $\Delta$ 's can be complex, too. Note that the unitary matrices have the form $U=R P O$, where only $P$ 's (given in (58)) are complex. Since $P$ 's are diagonal, they commute with $\mathbf{m}_{a L L, R R}^{2}$, so that $\Delta_{L L, R R}^{a}$ have no imaginary part. Further $\mathbf{m}^{\mathbf{2}}{ }_{a L R}$ has the same phase structure as the corresponding fermion mass matrix $\mathbf{m}^{a}$, which can be made real according to (57)- (61). Therefore, $\Delta_{L R}^{a}$, too, are real. Consequently, there is no $\mathrm{CP}$ violation originating from the SSB sector. The stringent constraints on $\Delta$ 's (80) coming from the EDMs [58, 59, 61] are automatically satisfied in this way of phase alignment. ${ }^{9}$

\footnotetext{
9 This does not mean that there is no CP violation in the SSB sector. Due to the existence of the multiple Higgs fields, there are one-loop diagrams contributing to the EDMs, even if all the SSB parameters are
} 


\section{2. $F C N C$}

In Refs. [56, 57], [26]-[31] [58]-[61], experimental bounds on the dimensionless quantities

$$
\delta_{L L, R R, L R}^{a}=\Delta_{L L, R R, L R}^{a} / m_{\tilde{a}}^{2} \quad(a=u, d),
$$

are given. The theoretical values of $\delta$ 's for the present model have been calculated in Ref. [22] as a function of the average sfermion masses and fine tuning parameters. The results may be summarized as follows. For the slepton sector where the average slepton mass $m_{\tilde{e}}$ is assumed to be $500 \mathrm{GeV}$, the theoretical values of $\left(\delta_{i j}^{\ell}\right)_{L L, R R, L R}$, except for $\left(\delta_{12}^{\ell}\right)_{L L}$, are several orders of magnitude smaller than the current experimental bounds, while $\left(\delta_{12}^{\ell}\right)_{L L}$ is of the same order as that of the experimental bound which comes from $\mu \rightarrow e \gamma$. In the squark sector, we find:

\section{Up quark sector:}

$$
\begin{aligned}
& \left(\delta_{12}^{u}\right)_{L L}=\left(\delta_{21}^{u}\right)_{L L} \simeq-1.5 \times 10^{-4} \Delta a_{L}^{q}, \\
& \left(\delta_{12}^{u}\right)_{R R}=\left(\delta_{21}^{u}\right)_{R R} \simeq-2.1 \times 10^{-4} \Delta a_{R}^{u}, \\
& \left(\delta_{12}^{u}\right)_{L R} \simeq-\left(\delta_{21}^{u}\right)_{L R} \simeq 6.2 \times 10^{-5}\left(-\tilde{A}_{a}^{u}+\tilde{A}_{b}^{u}+\tilde{A}_{b^{\prime}}^{u}-\tilde{A}_{c}^{u}\right)\left(\frac{500 \mathrm{GeV}}{m_{\tilde{q}}}\right),
\end{aligned}
$$

\section{$\underline{\text { Down quark sector }}$}

$$
\begin{aligned}
& \left(\delta_{12}^{d}\right)_{L L}=\left(\delta_{21}^{d}\right)_{L L} \simeq 2.2 \times 10^{-4} \Delta a_{L}^{q} \\
& \left(\delta_{13}^{d}\right)_{L L}=\left(\delta_{31}^{d}\right)_{L L} \simeq-8.1 \times 10^{-3} \Delta a_{L}^{q}, \\
& \left(\delta_{23}^{d}\right)_{L L}=\left(\delta_{32}^{d}\right)_{L L} \simeq 2.8 \times 10^{-2} \Delta a_{L}^{q} \\
& \left(\delta_{12}^{d}\right)_{R R}=\left(\delta_{21}^{d}\right)_{R R} \simeq-5.1 \times 10^{-2} \Delta a_{R}^{d}, \\
& \left(\delta_{13}^{d}\right)_{R R}=\left(\delta_{31}^{d}\right)_{R R} \simeq-0.1 \Delta a_{R}^{d} \\
& \left(\delta_{23}^{d}\right)_{R R}=\left(\delta_{32}^{d}\right)_{R R} \simeq-0.4 \Delta a_{R}^{d}
\end{aligned}
$$

where

$$
\Delta a_{L}^{q}=a_{L}^{q}-b_{L}^{q}, \Delta a_{R}^{a}=a_{R}^{a}-b_{R}^{a}, \tilde{A}_{i}^{a}=\frac{A_{i}^{a}}{m_{\tilde{q}}}(a=u, d) .
$$

These parameters, $a_{L, R}$ and $\tilde{A}_{i}$, are free dimensionless parameters, so that they are $O(1)$ if we do not fine tune them. The most stringent constraint in the up-sector comes from $\Delta M_{D}$ [30, 31]:

$$
\Delta M_{D}=>\left|\left(\delta_{12}^{u}\right)_{L L}\right|,\left|\left(\delta_{12}^{u}\right)_{R R}\right| \lesssim 6 \times 10^{-2},\left|\left(\delta_{12}^{u}\right)_{L R}\right|,\left|\left(\delta_{21}^{u}\right)_{L R}\right| \lesssim 10^{-2}
$$

real. The diagrams typically contain the $b$ terms, and we find that in the case of the present model $b_{-}<<m_{H_{-}^{u, d}}^{2}, b_{H}<m_{\phi_{H}^{u, d}}^{2}$ (given in (50 )) should be satisfied to satisfy the experimental constraints. 
for $m_{\tilde{q}}=0.5 \mathrm{TeV}$. As we can see from (82) this constraint can be satisfied without a finetuning. As for the down-sector we have to satisfy the constraints coming from $\Delta M_{K}, \Delta M_{B_{s}}$ and $\Delta M_{B_{d}}[26,29]$ :

$$
\begin{gathered}
\Delta M_{K}=>\left|\left(\delta_{12}^{d}\right)_{L L}\right|,\left|\left(\delta_{12}^{d}\right)_{R R}\right|,\left|\left(\delta_{12}^{d}\right)_{L R}\right|,\left|\left(\delta_{21}^{d}\right)_{L R}\right| \lesssim 10^{-3} \\
\Delta M_{B_{d}}=>\left|\left(\delta_{13}^{d}\right)_{L L}\right|,\left|\left(\delta_{13}^{d}\right)_{R R}\right|,\left|\left(\delta_{13}^{d}\right)_{L R}\right|,\left|\left(\delta_{31}^{d}\right)_{L R}\right| \lesssim 10^{-2} \\
\Delta M_{B_{s}}=>\left|\left(\delta_{23}^{d}\right)_{L L}\right|,\left|\left(\delta_{23}^{d}\right)_{R R}\right|,\left|\left(\delta_{23}^{d}\right)_{L R}\right|,\left|\left(\delta_{32}^{d}\right)_{L R}\right| \lesssim 10^{-1}
\end{gathered}
$$

Comparing these constraints with (83) we see that $\Delta a_{R}^{d}$ should be fine tuned at the level of few \%. ${ }^{10}$ In the next subsections we assume that $\Delta a_{R}^{d}$ is so small that only the heavy flavor-changing-neutral Higgs fields contribute to the mass differences of the neutral mesons.

\section{Flavor changing neutral Higgs couplings}

In Sec. IV we found that only the Higgs fields $\phi_{H,-}^{u, d}$ have flavor changing neutral couplings to the fermions, and that they have a definite form of mixing (see (49)). These are consequences of the $Z_{2}$ symmetry which is a part of the $O(2)$ flavor symmetry in the $\mu$ sector (as discussed in Sec. III. B). In the basis of the fermion mass eigenstates these Higgs couplings have the following form:

$$
\begin{aligned}
\mathcal{L}_{F C N C}= & -\left[Y_{i j}^{u H} \phi_{H}^{u}+Y_{i j}^{u-} \phi_{-}^{u}\right]^{*} \bar{u}_{i L}^{\prime} u_{j R}^{\prime}-\left[Y_{i j}^{d H} \phi_{H}^{d}+Y_{i j}^{d-} \phi_{-}^{d}\right]^{*} \bar{d}_{i L}^{\prime} d_{j R}^{\prime} \\
& -\left[Y_{i j}^{e H} \phi_{H}^{d}+Y_{i j}^{e-} \phi_{-}^{d}\right]^{*} \bar{e}_{i L}^{\prime} e_{j R}^{\prime}+\text { h.c. },
\end{aligned}
$$

where the Higgs fields are defined in (43), and

$$
\begin{aligned}
\mathbf{Y}^{u H} & =U_{L}^{u \dagger}\left[\frac{1}{\sqrt{2}} \cos \gamma^{u} e^{-i \theta_{+}^{u}}\left(\mathbf{Y}^{u 1}+\mathbf{Y}^{u 2}\right)-\sin \gamma^{u} e^{-i \theta_{3}^{u}} \mathbf{Y}^{u 3}\right] U_{R}^{u} \\
& =O_{L}^{u \dagger}\left[\frac{1}{\sqrt{2}} \cos \gamma^{u}\left(\mathbf{Y}^{u 1}+\mathbf{Y}^{u 2}\right)-\sin \gamma^{u} \mathbf{Y}^{u 3}\right] O_{R}^{u} \\
\mathbf{Y}^{d H} & =U_{L}^{d \dagger}\left[\frac{1}{\sqrt{2}} \cos \gamma^{d} e^{-i \theta_{+}^{d}}\left(\mathbf{Y}^{d 1}+\mathbf{Y}^{d 2}\right)-\sin \gamma^{d} e^{-i \theta_{3}^{d}} \mathbf{Y}^{d 3}\right] U_{R}^{d} \\
& =O_{L}^{d \dagger}\left[\frac{1}{\sqrt{2}} \cos \gamma^{d}\left(\mathbf{Y}^{d 1}+\mathbf{Y}^{d 2}\right)-\sin \gamma^{d} \mathbf{Y}^{d 3}\right] O_{R}^{d}, \\
\mathbf{Y}^{I-} & =U_{L}^{I \dagger}\left[\frac{1}{\sqrt{2}}\left(\mathbf{Y}^{I 1}-\mathbf{Y}^{I 2}\right)\right] U_{R}^{I}(I=u, d) .
\end{aligned}
$$

The Yukawa matrices $\mathbf{Y}^{u 1}$ etc. are given in (20), and the unitary matrices are given in (57)(61) and (72)-(75).

\footnotetext{
${ }^{10}$ We find that, as in the case of $\left(\delta_{12}^{u}\right)_{L R}$ of $(82)$, the left-right insertions $\left|\left(\delta_{12,21,13,31,23,32}^{d}\right)_{L R}\right|$ are much
} smaller than these constraints. 
The present model is consistent with the experimental observations in a certain region in the parameter space of the Yukawa couplings. An example of the choice of the nine parameters is given in (67), where we emphasize that this set of the nine parameters describe 10 physical independent quantities of the SM; six quark masses and four CKM parameters. Therefore, the consistent region in the space of the Yukawa couplings is very restricted, and we will be using only this set of the parameter values in the following discussion. Accordingly, for the values given in (67) we find the actual size of the Yukawa couplings:

$$
\begin{aligned}
Y_{a}^{u} & =\frac{\sqrt{2} m_{t} y_{u}^{2}}{v_{u} \cos \gamma^{u}} \simeq \frac{0.9957}{\sin \beta \cos \gamma^{u}}, Y_{b}^{u}=\frac{\sqrt{2} m_{t} b_{u}}{v_{u} \sin \gamma^{u}} \simeq \frac{0.05979}{\sin \beta \sin \gamma^{u}}, \\
Y_{b^{\prime}}^{u} & =\frac{\sqrt{2} m_{t} b_{u}^{\prime}}{v_{u} \sin \gamma^{u}} \simeq \frac{0.07054}{\sin \beta \sin \gamma^{u}}, Y_{c}^{u}=\frac{\sqrt{2} m_{t} q_{u}}{y_{u} v_{u} \cos \gamma^{u}} \simeq \frac{1.802 \times 10^{-4}}{\sin \beta \cos \gamma^{u}}, \\
Y_{a}^{d} & =\frac{\sqrt{2} m_{b} y_{d}^{2}}{v_{d} \cos \gamma^{d}} \simeq \frac{0.01478}{\cos \beta \cos \gamma^{d}}, Y_{b}^{d}=\frac{\sqrt{2} m_{b} b_{d}}{v_{d} \sin \gamma^{d}} \simeq \frac{5.449 \times 10^{-4}}{\cos \beta \sin \gamma^{d}}, \\
Y_{b^{\prime}}^{d} & =\frac{\sqrt{2} m_{b} b_{d}^{\prime}}{v_{d} \sin \gamma^{d}} \simeq \frac{7.702 \times 10^{-3}}{\cos \beta \sin \gamma^{d}}, Y_{c}^{d}=\frac{\sqrt{2} m_{b} q_{d}}{y_{d} v_{d} \cos \gamma^{d}} \simeq \frac{-6.701 \times 10^{-5}}{\cos \beta \cos \gamma^{d}},
\end{aligned}
$$

where $\gamma^{\prime}$ s and $\beta$ are given in (44) and (48), respectively, and we have used: $m_{t}=174 \mathrm{GeV}$, $m_{b}=2.9 \mathrm{GeV}$ and $v=\sqrt{v_{u}^{2}+v_{d}^{2}}=246 \mathrm{GeV}$. These parameters are defined in the $\overline{\mathrm{MS}}$ scheme and evaluated at the scale $M_{Z}$. With these numerical values we then obtain:

$$
\begin{aligned}
& \mathbf{Y}^{u H} \simeq \frac{1}{\tan \gamma^{u} \sin \beta}\left(\begin{array}{ccc}
-2.65 \times 10^{-4} & 3.22 \times 10^{-3} & 0.0439 \\
-3.22 \times 10^{-3} & 5.68 \times 10^{-3} & 0.0400 \\
0.0519 & -0.0473 & 6.02 \times 10^{-3}
\end{array}\right) \\
& -\frac{\tan \gamma^{u}}{\sin \beta}\left(\begin{array}{ccc}
7.63 \times 10^{-6} & -3.58 \times 10^{-4} & -2.52 \times 10^{-3} \\
-1.54 \times 10^{-6} & -4.17 \times 10^{-3} & -0.0592 \\
-2.99 \times 10^{-3} & 0.0699 & 0.991
\end{array}\right) \text {, } \\
& \mathbf{Y}^{u-} \simeq \frac{\exp i\left(2 \theta_{3}^{u}-\theta_{+}^{u}\right)}{\sin \gamma^{u} \sin \beta}\left(\begin{array}{ccc}
0 & -4.21 \times 10^{-3} & -0.0596 \\
-4.21 \times 10^{-3} & 0 & 2.54 \times 10^{-3} \\
0.0704 & 3.00 \times 10^{-3} & 0
\end{array}\right) \\
& \mathbf{Y}^{d H} \simeq \frac{1}{\tan \gamma^{d} \cos \beta}\left(\begin{array}{ccc}
6.63 \times 10^{-5} & 8.26 \times 10^{-5} & 2.80 \times 10^{-4} \\
-6.224 \times 10^{-5} & 3.74 \times 10^{-4} & 3.37 \times 10^{-4} \\
4.10 \times 10^{-3} & -6.01 \times 10^{-3} & 2.52 \times 10^{-3}
\end{array}\right) \\
& -\frac{\tan \gamma^{d}}{\cos \beta}\left(\begin{array}{ccc}
1.37 \times 10^{-5} & 1.13 \times 10^{-4} & 7.56 \times 10^{-5} \\
1.98 \times 10^{-5} & -1.88 \times 10^{-4} & -3.72 \times 10^{-4} \\
1.67 \times 10^{-3} & 6.61 \times 10^{-3} & 0.0131
\end{array}\right) \text {, } \\
& \mathbf{Y}^{d-} \simeq \frac{\exp i\left(2 \theta_{3}^{u}-\theta_{+}^{u}\right)}{\sin \gamma^{d} \cos \beta}\left(\begin{array}{ccc}
0 & -2.53 \times 10^{-4} & -4.72 \times 10^{-4} \\
-2.22 \times 10^{-4} & 0 & -1.04 \times 10^{-4} \\
7.46 \times 10^{-3} & -1.89 \times 10^{-3} & 0
\end{array}\right)
\end{aligned}
$$


The phases appearing in the matrices are given in (55) and (56). As we can see from these Yukawa matrices the size of the entries is fixed once the ratios of the VEVs $\left(\sin \beta, \sin \gamma^{u}\right.$ etc.) are fixed. For the down-type Yukawa matrices (99) and (100), for instance, all the entries (except the $(3,3)$ entry) are at most $O\left(10^{-3}\right)$. All these facts originate from the flavor symmetries of the model. Needless to say that in multi-Higgs models without a flavor symmetry this situation is completely different.

\section{FCNC}

The most severe FCNC constraints on the theory come from the mass differences in the neutral meson systems; $\Delta M_{D}, \Delta M_{K}, \Delta M_{B_{s}}$ and $\Delta M_{B_{d}}$. ${ }^{11}$ The Yukawa interaction terms that contribute to them can be found from (89):

$$
\begin{aligned}
\mathcal{L}_{\Delta M_{B}} & =-\left[Y_{u c}^{u H} \phi_{H}^{u}+Y_{u c}^{u-} \phi_{-}^{u}\right]^{*} \bar{u}_{L} c_{R}-\left[Y_{c u}^{u H} \phi_{H}^{u}+Y_{c u}^{u-} \phi_{-}^{u}\right] \bar{u}_{R} c_{L} \\
& -\left[Y_{s d}^{d H} \phi_{H}^{d}+Y_{s d}^{d-} \phi_{-}^{d}\right]^{*} \bar{s}_{L} d_{R}-\left[Y_{d s}^{d H} \phi_{H}^{d}+Y_{d s}^{d-} \phi_{-}^{d}\right] \bar{s}_{R} b_{L} \\
& -\left[Y_{b d}^{d H} \phi_{H}^{d}+Y_{b d}^{d-} \phi_{-}^{d}\right]^{*} \bar{b}_{L} d_{R}-\left[Y_{d b}^{d H} \phi_{H}^{d}+Y_{d b}^{d-} \phi_{-}^{d}\right] \bar{b}_{R} d_{L} \\
& -\left[Y_{b s}^{d H} \phi_{H}^{d}+Y_{b s}^{d-} \phi_{-}^{d}\right]^{*} \bar{b}_{L} s_{R}-\left[Y_{s b}^{d H} \phi_{H}^{d}+Y_{s b}^{d-} \phi_{-}^{d}\right] \bar{b}_{R} s_{L},
\end{aligned}
$$

where the values of the Yukawa couplings can be read off from (97)-(100). In (101) we have dropped the prime on the fields, which was indicating the mass eigenstate. As we can see from (51), no $\phi-\phi$ and $\phi^{*}-\phi^{*}$ type propagators contribute to the mass differences. So, only the $\phi-\phi^{*}$ type propagators can contribute, implying the phases in the Yukawa couplings (101) cancel in the tree-level diagrams contributing to the mass differences.

The independent parameters entering into $\Delta M_{D}$ are:

$$
\sin \beta, \sin \gamma^{u},\left(M_{H}^{u}\right)^{2}=\frac{\left(M_{H 1} M_{H 2}\right)^{2}}{m_{\phi_{H}^{d}}^{2}},\left(M_{-}^{u}\right)^{2}=\frac{\left(M_{-1} M_{-2}\right)^{2}}{m_{\phi_{-}^{d}}^{2}},
$$

where they are given, respectively, in (48), (44), (50), and (52). Similarly,

$$
\cos \beta, \sin \gamma^{d},\left(M_{H}^{d}\right)^{2}=\frac{\left(M_{H 1} M_{H 2}\right)^{2}}{m_{\phi_{H}^{u}}^{2}},\left(M_{-}^{d}\right)^{2}=\frac{\left(M_{-1} M_{-2}\right)^{2}}{m_{\phi_{-}^{u}}^{2}}
$$

enter into $\Delta M_{K}, \Delta M_{B_{s}}$ and $\Delta M_{B_{d}}$. With these remarks in mind, we proceed.

D1: Constraint from $\Delta M_{D}$

The following case is a special case of [31], and we will basically follow their analysis. An important difference here is that the size of all the Yukawa couplings is explicitly known.

11 The contribution to $\epsilon^{\prime} / \epsilon$ is negligibly small, at most $O\left(\left[10^{-7} / \alpha_{S}^{2}\right]\left[\tilde{m}_{q}^{2} / M^{2}\right]\right)$, where $\sim 10^{-7}$ originates from the Yukawa couplings relevant to this quantity, and $\tilde{m}_{q}$ and $M$ stand for the generic average squark and charged Higgs masses. See [62] and references therein for the constraint from the oblique corrections due to multiple $S U(2)_{L}$ doublet Higgs fields. 


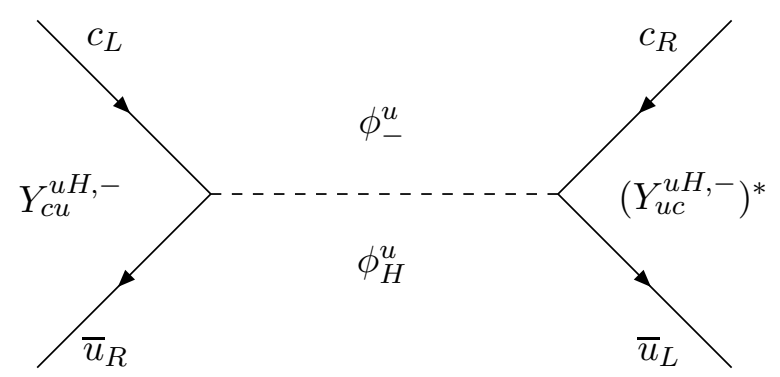

FIG. 1: The tree-diagram contributing to $\left(M_{D}^{\text {EXTRA }}\right)_{12}$. Tree-diagrams contributing to $M_{K}$ and $M_{B_{d, s}}$ are similar to this diagram. Leading QCD corrections [63] will be included, except for $\Delta M_{K}$.

The tree-level diagrams are shown in Fig. 1, As we can see from Fig. 1, only the $\bar{u}_{R} c_{L} \bar{u}_{L} c_{R}$ type operator contributes to $\Delta M_{D}$ at the tree-level. The mass difference $\Delta M_{D}$ can then be obtained from

$$
\Delta M_{D}=2\left|\left(M_{D}^{\mathrm{SM}}\right)_{12}+\left(M_{D}^{\mathrm{EXTRA}}\right)_{12}\right|
$$

where $\left(M_{D}^{\mathrm{SM}}\right)_{12}$ is the SM contribution, and

$$
\begin{aligned}
\left(M_{D}^{\text {EXTRA }}\right)_{12} & =2 C_{D}(\mu)<\bar{D}^{0}\left|\bar{u}_{R}^{\alpha} c_{L}^{\alpha} \bar{u}_{L}^{\beta} c_{R}^{\beta}\right| D^{0}>(\mu), \\
C_{D}(\mu) & =\eta(\mu)\left[\frac{Y_{c u}^{u H}\left(Y_{u c}^{u H}\right)^{*}}{\left(M_{H}^{u}\right)^{2}}+\frac{Y_{c u}^{u-}\left(Y_{u c}^{u-}\right)^{*}}{\left(M_{-}^{u}\right)^{2}}\right]
\end{aligned}
$$

with the QCD correction $\eta(\mu)$. The operator $\bar{u}_{R} c_{L} \bar{u}_{L} c_{R}$ can mix with $\bar{u}_{L} \gamma^{\mu} c_{L} \bar{u}_{R} \gamma_{\mu} c_{R}$ even at the leading order in QCD in principle [64]. However, if $\bar{u}_{L} \gamma^{\mu} c_{L} \bar{u}_{R} \gamma_{\mu} c_{R}$ is absent at $\mu=$ some energy, it will not be induced, at least in the leading order in QCD. Note that the values of the Yukawa matrices (97)-(100) are defined at $\mu=M_{Z}$, so that there are corrections if $\mu \neq M_{Z}$. We here take into account only QCD corrections because they are most dominant. The leading-order QCD correction $\eta$ takes the form [64]

$$
\begin{aligned}
\eta\left(\mu_{c}=2.8 \mathrm{GeV}\right) & =\left[\frac{\alpha_{s}\left(m_{b}\right)}{\alpha_{s}\left(\mu_{c}\right)}\right]^{-24 / 25}\left[\frac{\alpha_{s}\left(m_{t}\right)}{\alpha_{s}\left(m_{b}\right)}\right]^{-24 / 23}\left[\frac{\alpha_{s}(M)}{\alpha_{s}\left(m_{t}\right)}\right]^{-8 / 7}\left[\frac{\alpha_{s}\left(M_{Z}\right)}{\alpha_{s}(M)}\right]^{-8 / 7} \\
& \simeq 2.3
\end{aligned}
$$

where we have used the two-loop running of $\alpha_{s}(\mu)$ with $\alpha_{s}\left(M_{Z}\right)=0.119$, and the last factor is the QCD correction to the Yukawa matrices. So, the $M$ (which is supposed to be of the order of the heavy Higgs masses) dependence cancels nicely. The matrix element in the vacuum saturation approximation is given by [26]

$$
\begin{aligned}
<\bar{D}^{0}\left|\bar{u}_{R}^{\alpha} c_{L}^{\alpha} \bar{u}_{L}^{\beta} c_{R}^{\beta}\right| D^{0}>\left(\mu_{c}=2.8 \mathrm{GeV}\right) & =\frac{1}{4} f_{D}^{2} B_{D}^{\prime} M_{D}\left(\frac{M_{D}}{m_{c}}\right)^{2} \\
& \simeq 3.1 \times 10^{-2} \mathrm{GeV}^{3}
\end{aligned}
$$




\begin{tabular}{|c|c|c|c|}
\hline Input & & Input & \\
\hline$f_{D}$ & $\left(222.6 \pm 16.7_{-3.4}^{+2.8}\right) \times 10^{-3} \mathrm{GeV}$ & $B_{D}^{\prime}(2.8 \mathrm{GeV})$ & $1.08 \pm 0.03$ \\
\hline$M_{D}$ & $1.8645 \pm 0.0004 \mathrm{GeV}$ & $\tau_{D}$ & $(410.1 \pm 1.5) \times 10^{-3} \mathrm{ps}$ \\
\hline$x_{D}$ & $(5.3-11.7) \times 10^{-3}$ & $f_{K}$ & $(159.8 \pm 1.4 \pm 0.44) \times 10^{-3} \mathrm{GeV}$ \\
\hline$f_{B_{s}}$ & $\begin{array}{l}\text { I: } 0.240 \pm 0.040 \\
\text { II: } 0.245 \pm 0.013\end{array}$ & $B_{s}^{\prime}\left(m_{b}\right)$ & $1.16 \pm 0.02 \underset{-0.07}{+0.05}$ \\
\hline$f_{B_{s}} \sqrt{B_{s}}$ & $\begin{array}{l}\text { I: } 0.221 \pm 0.046 \\
\text { II: } 0.227 \pm 0.017\end{array}$ & $\xi$ & $1.24 \pm 0.04$ \\
\hline$f_{B_{d}}$ & $0.198 \pm 0.017 \mathrm{GeV}$ & $B_{d}^{\prime}\left(m_{b}\right)$ & $1.15 \pm 0.03 \stackrel{+0.05}{+0.07}$ \\
\hline$M_{K}$ & $0.497648 \pm 0.000022 \mathrm{GeV}$ & $\Delta M_{K}^{\exp }$ & $(0.5292 \pm 0.0009) \times 10^{-2} \mathrm{ps}^{-1}$ \\
\hline$M_{B_{s}}$ & $5.3661 \pm 0.0006 \mathrm{GeV}$ & $\Delta M_{B_{s}}^{\exp }$ & $17.77 \pm 0.10 \pm 0.07 \mathrm{ps}^{-1}$ \\
\hline$M_{B_{d}}$ & $5.27950 \pm 0.00033 \mathrm{GeV}$ & $\Delta M_{B_{d}}^{\exp }$ & $0.507 \pm 0.005 \mathrm{ps}^{-1}$ \\
\hline$m_{u}(2 \mathrm{GeV})$ & $(3 \pm 1) \times 10^{-3} \mathrm{GeV}$ & $m_{c}\left(m_{c}\right)$ & $1.30 \pm 0.05 \mathrm{GeV}$ \\
\hline$m_{d}(2 \mathrm{GeV})$ & $(6.0 \pm 1.5) \times 10^{-3} \mathrm{GeV}$ & $m_{s}(2 \mathrm{GeV})$ & $0.10 \pm 0.02 \mathrm{GeV}$ \\
\hline$m_{d}\left(m_{b}\right)$ & $(5.1 \pm 1.3) \times 10^{-3} \mathrm{GeV}$ & $m_{s}\left(m_{b}\right)$ & $0.085 \pm 0.017 \mathrm{GeV}$ \\
\hline$m_{t}\left(m_{t}\right)$ & $163.8 \pm 2.0 \mathrm{GeV}$ & $m_{b}\left(m_{b}\right)$ & $4.22 \pm 0.08 \mathrm{GeV}$ \\
\hline
\end{tabular}

TABLE III: Parameter values used in the text (see also Ref. [52]). $f_{D}$ is taken from [65], and we use $B_{D}^{\prime}$ and $x_{D}$ of [30] and [66], respectively. $M_{D}, \tau_{D}, f_{K}, M_{K}, \Delta M_{K}^{\exp }, M_{B_{s}}, M_{B_{d}}, \Delta M_{B_{d}}^{\exp }$ are from [51]. $f_{B_{s}}$ (I) and $f_{B_{s}} \sqrt{B_{s}}$ (I) are the conservative sets of [72], and $f_{B_{s}} \sqrt{B_{s}}$ (II) is found in [67], while $f_{B_{s}}$ (II) and $\xi$ are taken from [68], and $f_{B_{d}}$ is obtained from $f_{B_{s}} / \xi$. (See [69] for a more conservative estimate of $\xi$, and references therein.) $B_{s}^{\prime}$ and $B_{d}^{\prime}$ are found in [70]. $\Delta M_{B_{s}}^{\exp }$ is from [71]. $m_{u}(2 \mathrm{GeV})$ and $m_{d}(2 \mathrm{GeV})$ are from [51], while the mass values of the other quarks are taken from [72], in which the relevant references are given.

where we have used the central values of the parameters ${ }^{12}$ given in Table III. $\left(m_{c}(2.8 \mathrm{GeV})=\right.$ $1.0 \mathrm{GeV}$ which corresponds to $m_{c}\left(m_{c}\right)=1.3 \mathrm{GeV}$.)

Clearly, the larger $\left(M_{H}^{u}\right)^{2}$ and $\left(M_{-}^{u}\right)^{2}$ are, the smaller are the extra contributions. Here we are interested in the minimal values of $\left(M_{H}^{d}\right)^{2}$ and $\left(M_{-}^{d}\right)^{2}$, which are consistent with the observations. We find that the Wilson coefficient $C_{D}$ becomes

$$
\begin{aligned}
C_{D}\left(\mu_{c}\right) & =\frac{\eta\left(\mu_{c}\right)}{\sin ^{2} \beta}\left[\frac{1 \mathrm{TeV}}{M_{H}^{u}}\right]^{2} \times 10^{-11} \\
& \times\left(\frac{1.772}{r_{u}^{2} \sin ^{2} \gamma^{u}}-\frac{1.037}{\tan ^{2} \gamma^{u}}-0.115+5.5 \times 10^{-5} \tan ^{2} \gamma^{u}\right) \mathrm{GeV}^{-2}
\end{aligned}
$$

${ }^{12}$ Since we take here a conservative standpoint that the extra contribution can be as large as the experimental value, we ignore the details of uncertainties. 
where

$$
r_{u}=\frac{M_{-}^{u}}{M_{H}^{u}}=\left(\frac{M_{-1} M_{-2}}{M_{H 1} M_{H 2}}\right) \quad\left(\frac{m_{\phi_{H}^{d}}}{m_{\phi_{-}^{d}}}\right),
$$

and the mass parameters are defined in (102). If each term in (110) should satisfy the constraint,

$$
\left|\Delta M_{D}^{\mathrm{EXTRA}}\right|=2\left|\left(M_{D}^{\mathrm{EXTRA}}\right)_{12}\right|<\Delta M_{D}^{\mathrm{exp}}=x_{D} / \tau_{D} \simeq 1.4 \times 10^{-14} \mathrm{GeV},
$$

one finds that $\sin \beta M_{H}^{u} \gtrsim 17 \mathrm{TeV}$ and $\sin \beta M_{-}^{u} \gtrsim 22 \mathrm{TeV}$ should be satisfied. We, however, observe that the terms in (110) can cancel each other, so that no lower bounds on $M_{H}^{u}$ and $M_{-}^{u}$ can be obtained. In Fig. 2 we show the region in the $\sin \gamma^{u}-r_{u}$ plane for $\sin \beta M_{H}^{u}=2$ $\mathrm{TeV}$, in which $\left|\Delta M_{D}^{\mathrm{EXTRA}}\right|$ is smaller than the smallest $\Delta M_{D}^{\mathrm{exp}}$, i.e.,

$$
\left|\Delta M_{D}^{\mathrm{EXTRA}}\right|<8 \times 10^{-15} \mathrm{GeV}
$$

We see from Fig. 2 that to satisfy the constraint (113), we have to fine tune $r_{u}$ and $\sin \gamma^{u}$

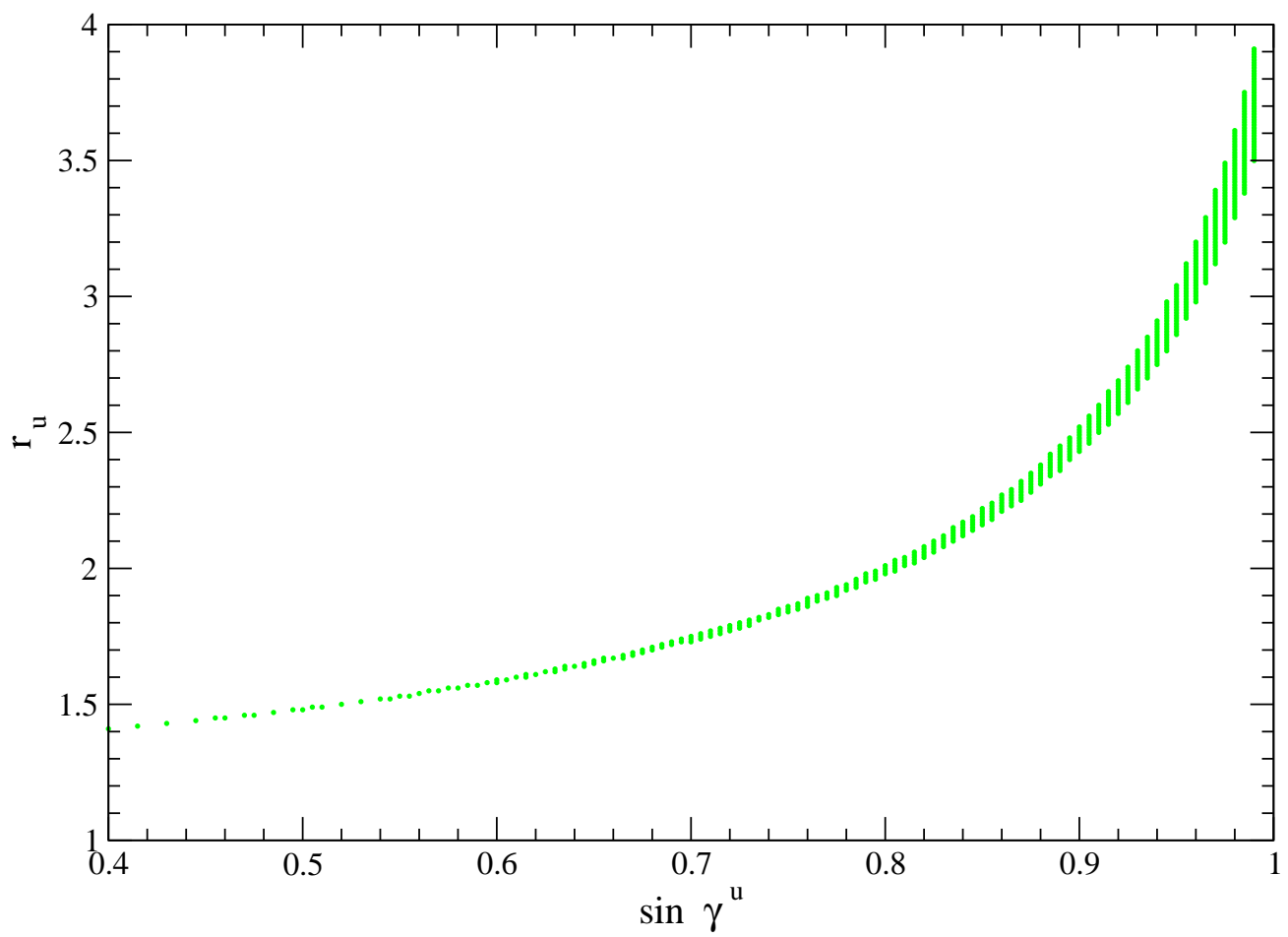

FIG. 2: The region in the $\sin \gamma^{u}-r_{u}$ plane, in which the constraint (113) coming from $\Delta M_{D}$ is satisfied for $\sin \beta M_{H}^{u}=2 \mathrm{TeV}$, where $r_{u}, \sin \gamma^{u}$ and $M_{H}^{u}$ are defined in (111), (44) and (102), respectively.

even for $\sin \beta M_{H}^{u}=2 \mathrm{TeV}$.

The neutral Higgs bosons in question can induce processes such as $D^{0} \rightarrow e^{+} e^{-}$and $D^{0} \rightarrow$ $\mu^{+} \mu^{-}$which are strongly suppressed. The experimental upper bounds of the branching ratios 
are smaller than $O\left(10^{-6}\right)$. From a rough estimate we find that $M_{-}^{u}, M_{H}^{u}>M_{Z}$ is more than sufficient to suppress these processes. So, in principle, $M_{-}^{u}, M_{H}^{u}$ could be light, although one needs an extreme fine tuning between $r_{u}$ and $\sin \gamma^{u}$.

D2: Constraint from $\Delta M_{K}$

As in the case of $\Delta M_{D}$, the interaction Lagrangian generates only one type of the $\Delta S=2$ operator at the tree level. So, the relevant matrix element is

$$
\begin{aligned}
<\bar{K}^{0}\left|\bar{s}_{R}^{\alpha} d_{L}^{\alpha} \bar{s}_{L}^{\beta} d_{R}^{\beta}\right| K^{0}> & =\frac{1}{4} f_{B_{K}}^{2} B_{K}^{\prime} M_{K}\left(\frac{M_{K}}{m_{s}+m_{d}}\right)^{2} \\
& \simeq 0.28 \mathrm{GeV}^{3}
\end{aligned}
$$

where we have used the central values of the parameters given in Table III. (As in the case of $\Delta M_{D}$ we we ignore the details of uncertainties involved in $\Delta M_{D}$.) As far as we understand, there is no reliable calculation of $B_{K}^{\prime}$ for the present case (114), ${ }^{13}$ and so we assume that $B_{K}^{\prime}=1$. Correspondingly, we do not take into account QCD corrections for the present case.

The tree-level coefficient is given by

$$
\begin{aligned}
C_{K} & =\left[\frac{Y_{d s}^{d H}\left(Y_{s d}^{d H}\right)^{*}}{\left(M_{H}^{d}\right)^{2}}+\frac{Y_{d s}^{d-}\left(Y_{s d}^{d-}\right)^{*}}{\left(M_{-}^{d}\right)^{2}}\right] \\
& =\frac{1}{\cos ^{2} \beta}\left[\frac{1 \mathrm{TeV}}{M_{H}^{d}}\right]^{2} \times 10^{-14} \\
& \times\left(\frac{5.617}{r_{d}^{2} \sin ^{2} \gamma^{d}}-\frac{0.514}{\tan ^{2} \gamma^{d}}-0.539+0.224 \tan ^{2} \gamma^{d}\right) \mathrm{GeV}^{-2}
\end{aligned}
$$

where

$$
r_{d}=\frac{M_{-}^{d}}{M_{H}^{d}}=\left(\frac{M_{-1} M_{-2}}{M_{H 1} M_{H 2}}\right) \quad\left(\frac{m_{\phi_{H}^{d}}}{m_{\phi_{-}^{d}}}\right) .
$$

In Fig. 3 we show the region in the $r_{d}-\sin \gamma^{d}$ plane in which

$$
\Delta M_{K}=2 \times 0.28 \times C_{K} \mathrm{GeV}<\Delta M_{K}^{\exp } \simeq 3.49 \times 10^{-15} \mathrm{GeV}
$$

is satisfied.

D3: Constraint from $\Delta M_{B_{s}}, \Delta M_{B_{d}}$

As in the previous cases, the mass differences can be obtained from

$$
\Delta M_{B_{s, d}}=2\left|<\bar{B}^{0}\right|\left(M_{s, d}^{\mathrm{SM}}\right)_{12}+\left(M_{s, d}^{\mathrm{EXTRA}}\right)_{12}\left|B^{0}>\right| .
$$

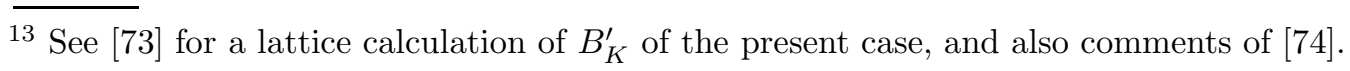




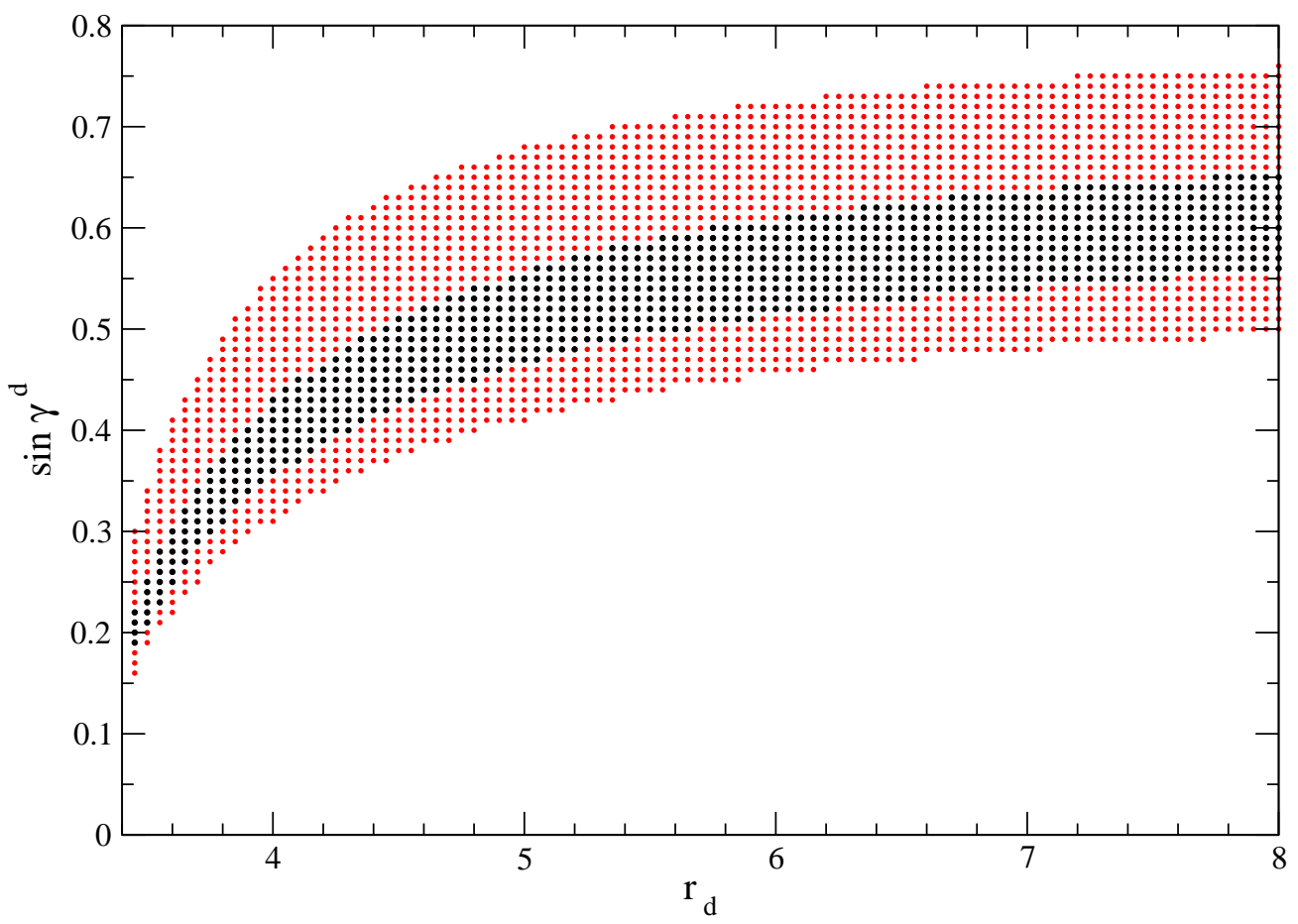

FIG. 3: The region in the $r_{d}-\sin \gamma^{d}$ plane for $\cos \beta M_{H}^{d}=0.5 \mathrm{TeV}$ (red (dark grey)) and $0.3 \mathrm{TeV}$ (black), in which $\left|\Delta M_{K}^{\mathrm{EXTRA}}\right|<\Delta M_{K}^{\exp }$ is satisfied. $r_{d}$ and $\sin \gamma^{d}$ are defined in (116) and (44), respectively.

The SM contributions to $\Delta M_{B_{s}}, \Delta M_{B_{d}}$ are well controlled up to the numerical uncertainty in the decay constants. Here following [72], which is based on the NLO-QCD calculations in Refs. [75] and [76], we consider two sets of the uncertainties for the $B$ system, I and II, as one can see in Table III. Since the uncertainties in the decay constants are much larger than those of other quantities, we assume that

$$
\begin{aligned}
& f_{B_{s}} \sqrt{B_{s}}=\left\{\begin{array}{c}
0.221 \pm 0.046 \text { for the parameter set I } \\
0.227 \pm 0.017 \text { for the parameter set II }
\end{array}\right. \\
& f_{B_{d}} \sqrt{B_{d}}=\left\{\begin{array}{l}
0.181 \pm 0.044 \text { for the parameter set I } \\
0.184 \pm 0.017 \text { for the parameter set II }
\end{array}\right.
\end{aligned}
$$

are the only uncertainties for the SM model contributions $M_{s, d}^{\mathrm{SM}}$, where $f_{B_{d}} \sqrt{B_{d}}$ is obtained from $\xi=f_{B_{s}} \sqrt{B_{s}} / f_{B_{d}} \sqrt{B_{d}}$. To simplify the situation further, we assume that this is also true for the extra contributions $M_{s, d}^{\mathrm{EXTRA}}$.

To calculate $\left(M_{s, d}^{\mathrm{SM}}\right)_{12}$ we use the parameter values (68) which are predicted in the present model:

$$
\left|V_{\mathrm{CKM}}\right|_{u s}=0.2266,\left|V_{\mathrm{CKM}}\right|_{u b}=0.00362,\left|V_{\mathrm{CKM}}\right|_{c b}=0.0417, \phi_{3}(\gamma)=1.107 .
$$


Then we follow the calculation of [72] and obtain:

$$
\begin{aligned}
2\left(M_{B_{s}}^{\mathrm{SM}}\right)_{12} & =2\left|\left(\bar{M}_{s}^{\mathrm{SM}}\right)_{12}\right|\left(1 \pm \delta_{s}\right) \exp i \phi_{s} \\
& =\left\{\begin{array} { l } 
{ 1 9 . 5 ( 1 \pm 0 . 4 6 ) \operatorname { e x p } ( - i 0 . 0 0 3 5 ) } \\
{ 2 0 . 6 ( 1 \pm 0 . 1 6 ) \operatorname { e x p } ( - i 0 . 0 0 3 5 ) }
\end{array} \mathrm { ps } ^ { - 1 } \text { for } \left\{\begin{array}{c}
\mathrm{I} \\
\mathrm{II}
\end{array},\right.\right. \\
2\left(M_{B_{s}}^{\mathrm{SM}}\right)_{12} & =2\left|\left(\bar{M}_{d}^{\mathrm{SM}}\right)_{12}\right|\left(1 \pm \delta_{d}\right) \exp i \phi_{d} \\
& =\left\{\begin{array} { l } 
{ 0 . 5 6 ( 1 \pm 0 . 5 5 ) \operatorname { e x p } ( i 0 . 7 7 ) } \\
{ 0 . 5 9 ( 1 \pm 0 . 1 9 ) \operatorname { e x p } ( i 0 . 7 7 ) }
\end{array} \mathrm { ps } ^ { - 1 } \text { for } \left\{\begin{array}{c}
\mathrm{I} \\
\mathrm{II}
\end{array}\right.\right.
\end{aligned}
$$

where $\left(\bar{M}_{s, d}^{\mathrm{SM}}\right)_{12}$ are the SM contributions which are obtained with the central values of $f_{B_{s}} \sqrt{B_{s}}, \xi, M_{B_{s, d}}$ and the quark masses given ${ }^{14}$ in III and $\alpha_{s}\left(M_{Z}\right)=0.119$, and $\delta_{s}$ and $\delta_{d}$ correspond to the uncertainties in $f_{B_{s}} \sqrt{B_{s}}$ and $f_{B_{d}} \sqrt{B_{d}}$ given in (120), respectively. As we can see from Table III, the SM values are slightly larger than the experimental values.

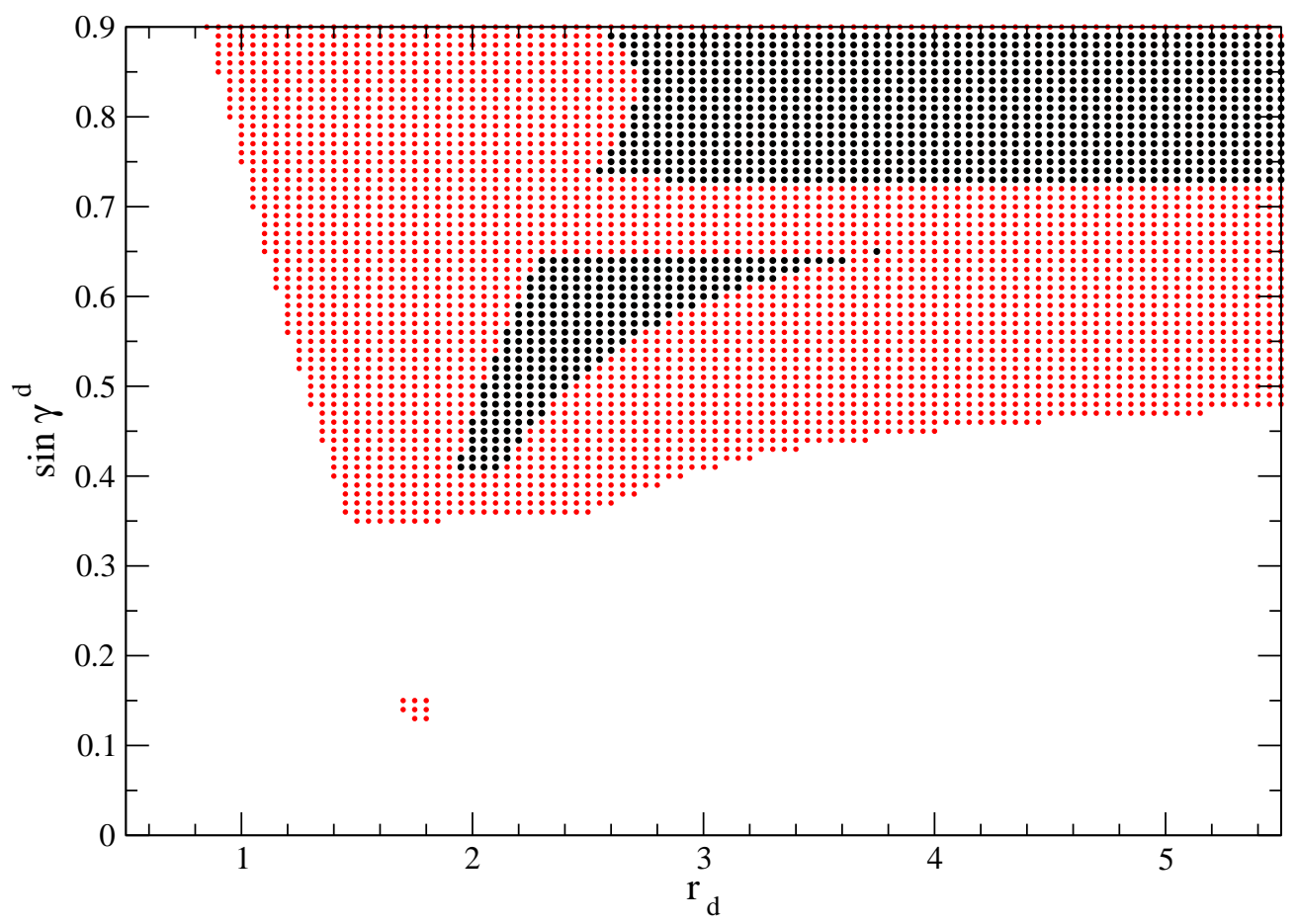

FIG. 4: The allowed region for the parameter set I with $\cos \beta M_{H}^{d}=0.50$ (black) and 1.5 (red (dark grey)) $\mathrm{TeV}$ in which the constraints (129) and (130) are simultaneously satisfied. $r_{d}$ and $\sin \gamma^{d}$ are defined in (116) and (44), respectively. Two sets of values I and II are given in Table III,

14 The model does not predict the absolute scale for the quark masses. If we use the mass ratio given in (68), we obtain a slightly smaller value for $m_{b}\left(m_{b}\right)$ (while we obtain the same value for $m_{c}\left(m_{c}\right)$ ). This difference has only a negligible effect on the SM contributions. 


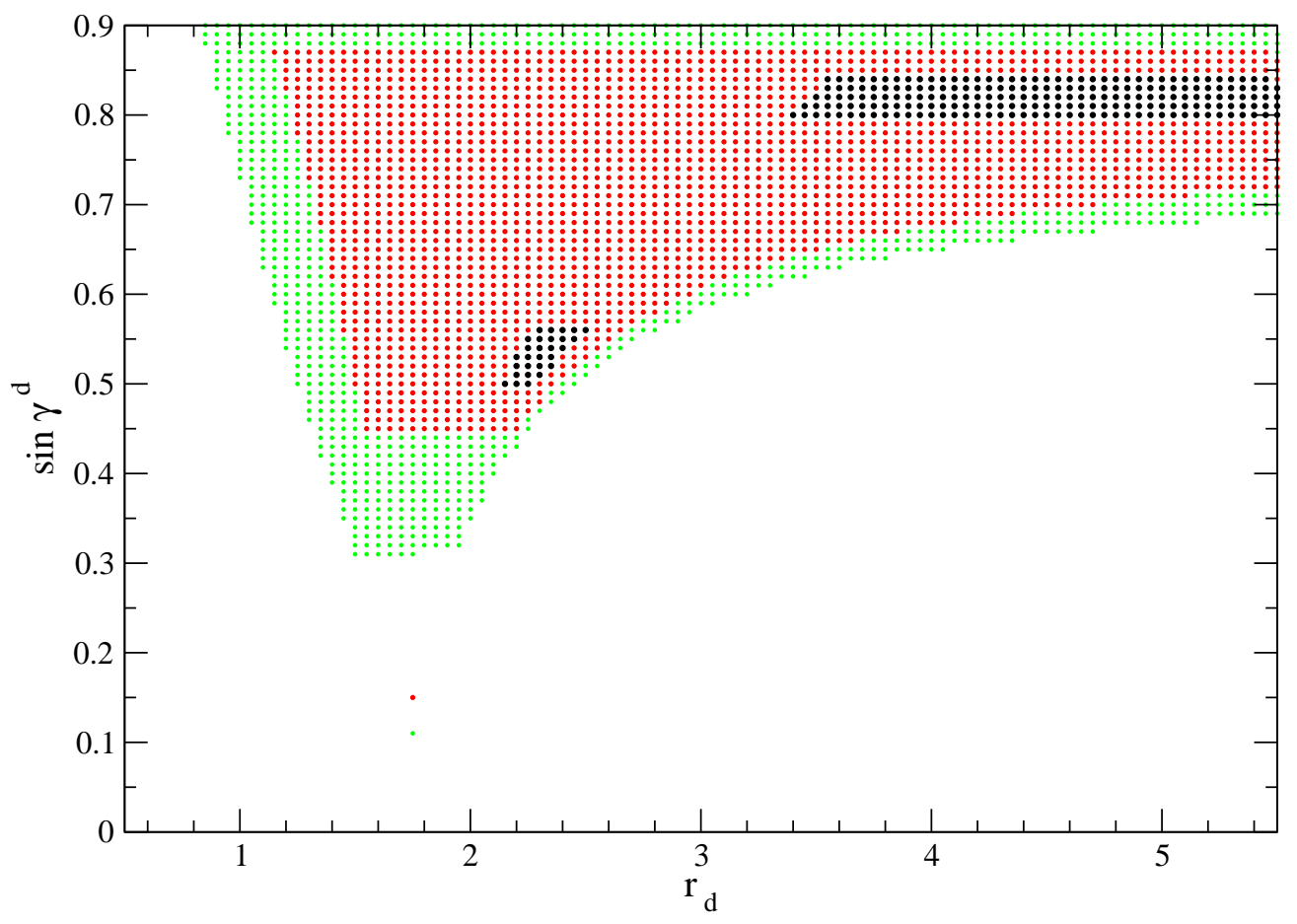

FIG. 5: The same as Fig. 廿 4 for the parameter set II with $\cos \beta M_{H}^{d}=0.50$ (black), 1.5 (red (dark grey)) and 2 (green (grey)) $\mathrm{TeV}$.

As for the extra contributions, only the matrix elements

$$
\begin{aligned}
<{\overline{B_{s}}}^{0}\left|\bar{b}_{R}^{\alpha} s_{L}^{\alpha} \bar{b}_{L}^{\beta} s_{R}^{\beta}\right| B_{s}^{0}> & =\frac{1}{4} f_{B_{s}}^{2} B_{s}^{\prime} M_{B_{s}}\left(\frac{M_{B_{s}}}{m_{b}+m_{s}}\right)^{2} \\
& \simeq\left\{\begin{array}{c}
0.29(\mathrm{I}) \\
0.30(\mathrm{II})
\end{array} \mathrm{GeV}^{3}\right.
\end{aligned}
$$

and

$$
\begin{aligned}
<{\overline{B_{d}}}^{0}\left|\bar{b}_{R}^{\alpha} d_{L}^{\alpha} \bar{b}_{L}^{\beta} d_{R}^{\beta}\right| B_{d}^{0}> & =\frac{1}{4} f_{B_{d}}^{2} B_{d}^{\prime} M_{B_{d}}\left(\frac{M_{B_{d}}}{m_{b}+m_{d}}\right)^{2} \\
& \simeq 0.18(\mathrm{I}, \mathrm{II}) \mathrm{GeV}^{3}
\end{aligned}
$$

are relevant for $\Delta M_{B_{s}}, \Delta M_{B_{d}}$, where the tree-level diagrams similar to Fig. 1 contribute to these mass differences, and we have used the central values of the parameters in Table III. 


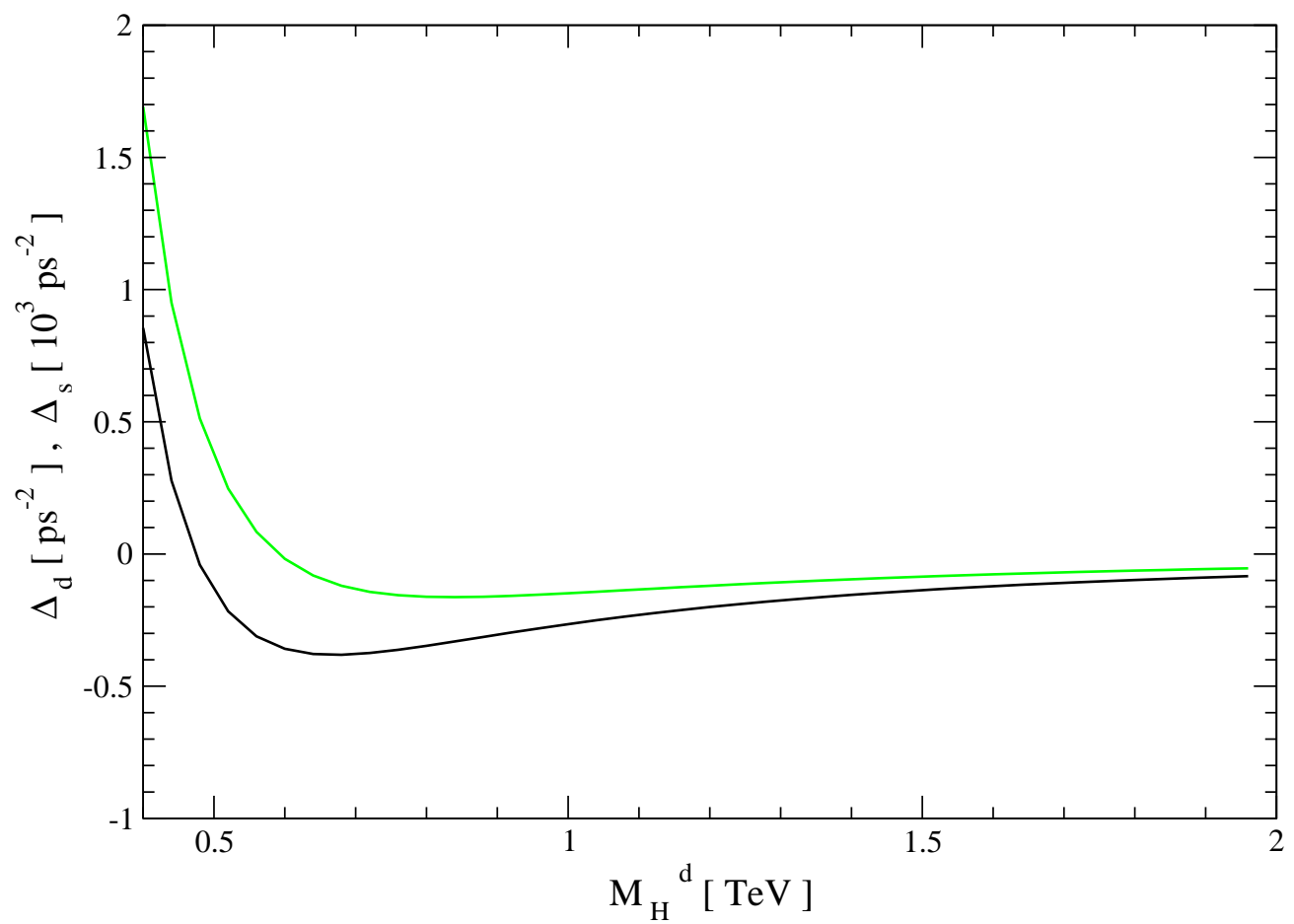

FIG. 6: $\Delta_{s}$ (green (grey)) and $\Delta_{d}$ (black) for the parameter set I with $r_{d}=3$ and $\sin \gamma^{d}=0.8$, where they are defined in (132). This graph explains why the allowed region in the $r_{d}-\sin \gamma^{d}$ plane first shrinks and then extends as $M_{H}^{d}$ increases. For the the parameter set II we obtain a similar result.

The leading order Wilson coefficients are

$$
\begin{aligned}
C_{B_{s}} & =\eta_{B}\left(m_{b}\right) \frac{1}{\cos ^{2} \beta}\left[\frac{1 \mathrm{TeV}}{M_{H}^{d}}\right]^{2} \times 10^{-12} \\
& \times\left(\frac{0.197}{r_{d}^{2} \sin ^{2} \gamma^{d}}-\frac{2.025}{\tan ^{2} \gamma^{d}}-4.463-2.459 \tan ^{2} \gamma^{d}\right) \mathrm{GeV}^{-2} \\
C_{B_{d}} & =\eta_{B}\left(m_{b}\right) \frac{1}{\cos ^{2} \beta}\left[\frac{1 \mathrm{TeV}}{M_{H}^{d}}\right]^{2} \times 10^{-12} \\
& \times\left(-\frac{3.521}{r_{d}^{2} \sin ^{2} \gamma^{d}}+\frac{1.148}{\tan ^{2} \gamma^{d}}-0.780+0.127 \tan ^{2} \gamma^{d}\right) \mathrm{GeV}^{-2},
\end{aligned}
$$

where

$$
\begin{aligned}
\eta_{B}\left(m_{b}=4.22 \mathrm{GeV}\right) & =\left[\frac{\alpha_{s}\left(m_{t}\right)}{\alpha_{s}\left(m_{b}\right)}\right]^{-24 / 23}\left[\frac{\alpha_{s}(M)}{\alpha_{s}\left(m_{t}\right)}\right]^{-8 / 7}\left[\frac{\alpha_{s}\left(M_{Z}\right)}{\alpha_{s}(M)}\right]^{-8 / 7} \\
& \simeq 2.0
\end{aligned}
$$

Then we require that

$$
\begin{aligned}
& \Delta M_{B_{s}}=\Delta M_{B_{s}}^{\exp }=2\left|\left(M_{B_{s}}^{\mathrm{SM}}\right)_{12}+0.41 \times C_{B_{s}}\right|=17.77 \mathrm{ps}^{-1}=1.17 \times 10^{-11} \mathrm{GeV} \\
& \Delta M_{B_{d}}=\Delta M_{B_{d}}^{\mathrm{exp}}=2\left|\left(M_{B_{d}}^{\mathrm{SM}}\right)_{12}+0.25 \times C_{B_{d}}\right|=0.507 \mathrm{ps}^{-1}=3.34 \times 10^{-13} \mathrm{GeV}
\end{aligned}
$$




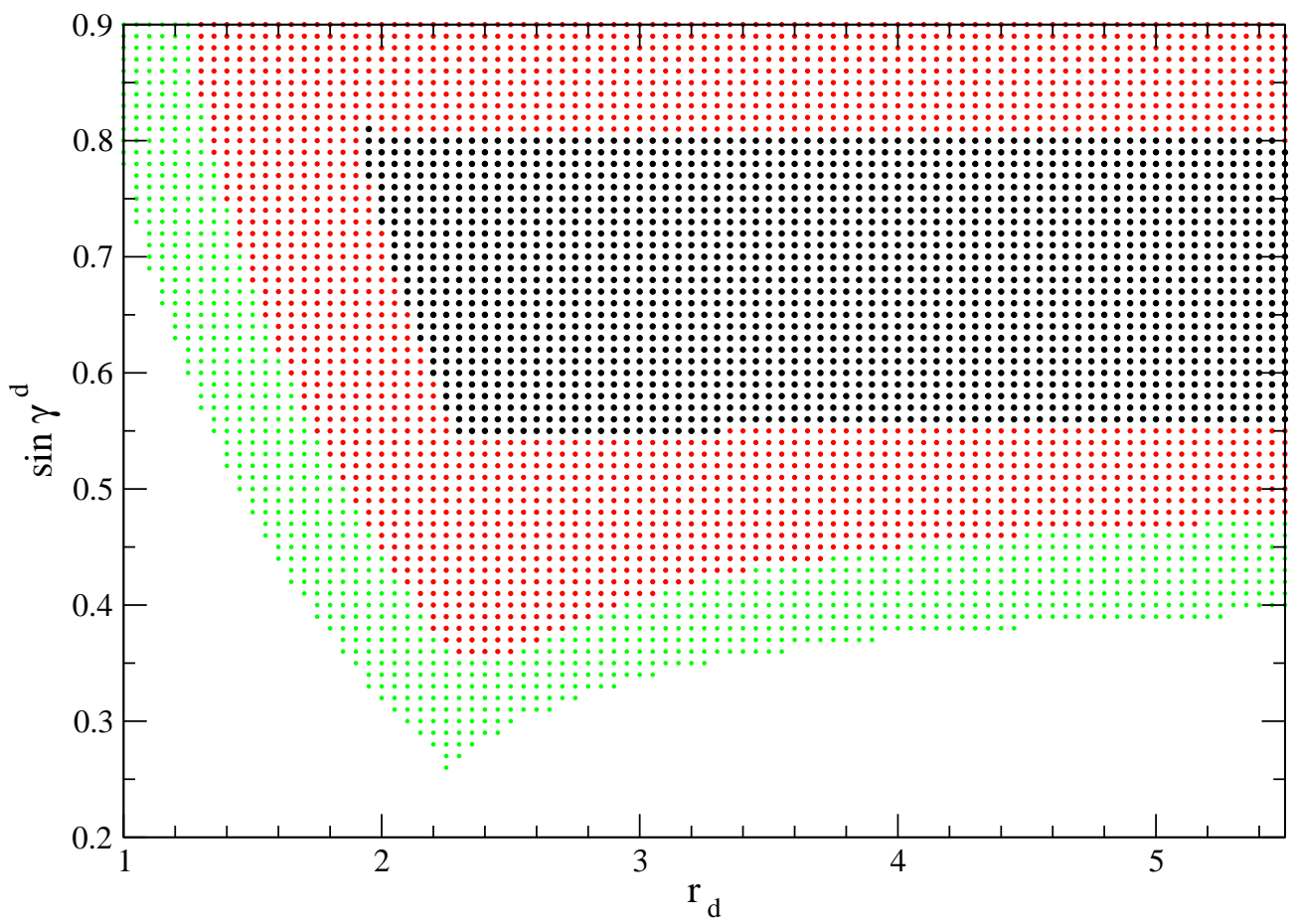

FIG. 7: The region in which the constraints (117), (129) and (130) coming from $\Delta M_{K}, \Delta M_{B_{s, d}}$ are satisfied for the parameter set I with $M_{H}^{d}=1.1$ (black), $M_{H}^{d}=1.5$ (red (dark grey)) and 2 (green (grey)) TeV. $r_{d}$ and $\sin \gamma^{d}$ are defined in (116) and (44), respectively.

Note that according to our assumption the uncertainties factorize as $\left(1 \pm \delta_{s, d}\right)\left[\left(\bar{M}_{s, d}^{\mathrm{SM}}\right)_{12}+\right.$ $\left.\left(M_{s, d}^{\text {EXTRA }}\right)_{12}\right]$, where $\bar{M}_{s, d}$ are the central values and $\delta_{s(d)}$ are given in (122) and (123). In Fig. 4 and 5 we show the allowed region in the $r_{d}-\sin \gamma^{d}$ plane for the parameter sets I and II, respectively, in which (129) and (130) are satisfied. We find that (129) and (130) can be simultaneously satisfied even for small $M_{H}^{d} \gtrsim 0.50 \mathrm{TeV}$. The allowed region shrinks as $M_{H}^{d}$ increases. At $M_{H}^{d}=1 \mathrm{TeV}$ the allowed region is very small. But a wide allowed region exists for $M_{H}^{d}=2 \mathrm{TeV}$. The reason that the allowed region first decreases and then increases as $M_{H}^{d}$ increases starting from $\simeq 0.50 \mathrm{TeV}$ is the following. The constraint (129) and and (130) can be written as

$$
\left[\Delta M_{B_{s, d}}^{\exp }\right]^{2}\left(1+\delta_{s}\right)^{-2} \leq \Delta_{s, d}+4\left[\left(\bar{M}_{s}^{\mathrm{SM}}\right)_{12}\right]^{2} \leq\left[\Delta M_{B_{s, d}}^{\exp }\right]^{2}\left(1-\delta_{s}\right)^{-2},
$$

where

$$
\Delta_{s, d}=4\left[\left(M_{s, d}^{\mathrm{EXTRA}}\right)_{12}\right]^{2}+8 \cos \phi_{s, d}\left(\bar{M}_{s, d}^{\mathrm{SM}}\right)_{12}\left(M_{s, d}^{\mathrm{EXTRA}}\right)_{12},
$$

and $\left(\bar{M}_{s, d}^{\mathrm{SM}}\right)_{12}$ and $\phi_{s, d}$ are given in (122) and (123). For a large $M_{H}^{d}$ the second term of $\Delta_{s, d}$ is dominant. However, for a small $M_{H}^{d}$, two terms can become of the same order, and since $\left(M_{B_{s}}^{\mathrm{EXTRA}}\right)_{12}$ and $\left(M_{B_{d}}^{\mathrm{EXTRA}}\right)_{12}$ can simultaneously become negative, these two terms can cancel each other, so that the both constraints (131) for $\Delta M_{B_{s}}$ and $\Delta M_{B_{d}}$ can be 


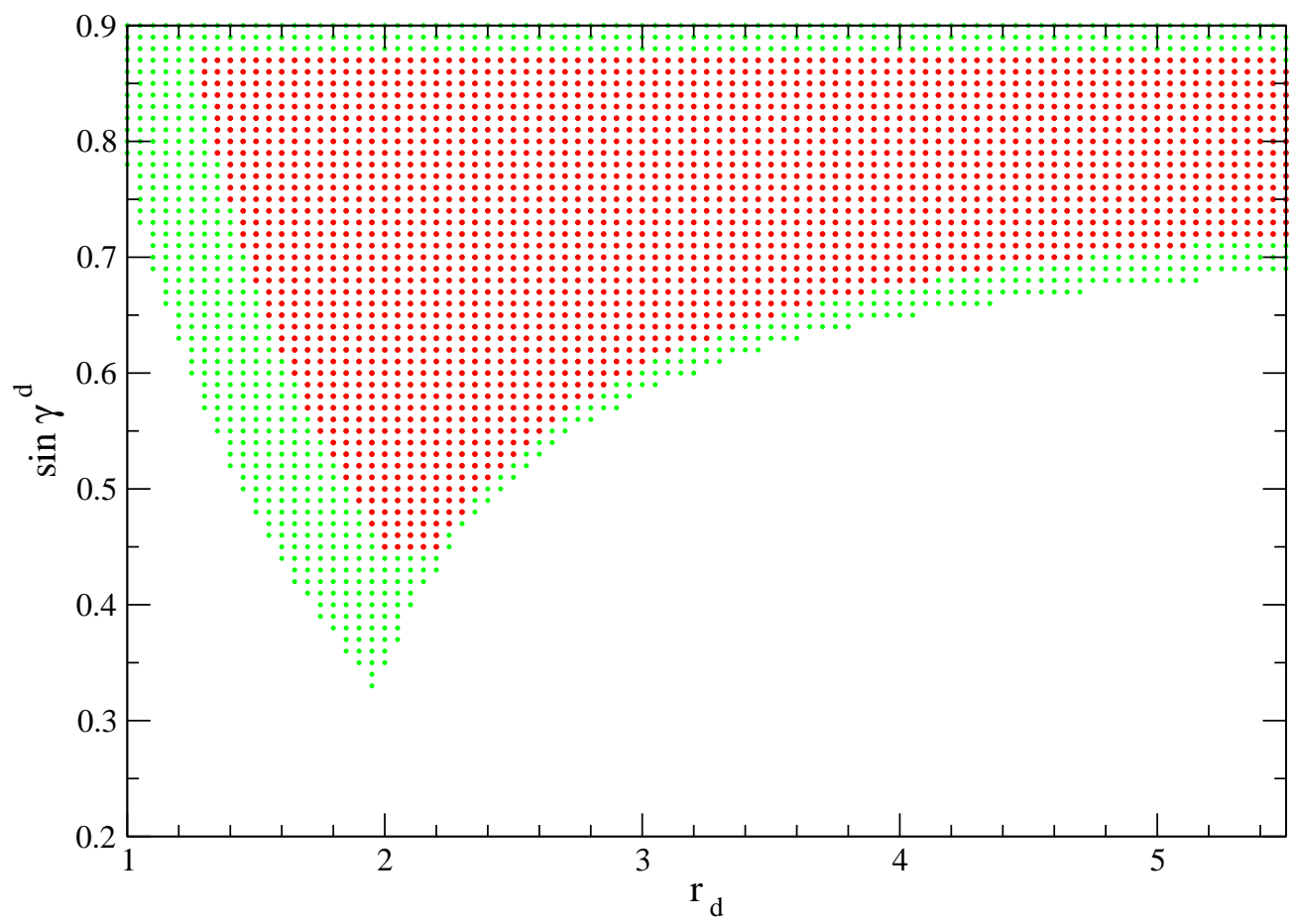

FIG. 8: The same as Fig. 7 for the parameter set II with $M_{H}^{d}=1.5$ (red (dark grey)) and 2 (green (grey)) $\mathrm{TeV}$.

simultaneously satisfied. In Fig. [6 we show $\Delta_{s}$ (red) and $\Delta_{d}$ (blue) for the parameter set I as a function of $M_{H}^{d}$ for $r_{d}=3$ and $\sin \gamma^{d}=0.8$, where we vary $M_{H}^{d}$ from $0.4 \mathrm{TeV}$ to 2 $\mathrm{TeV}$. We see from the figure that $\Delta_{s}$ and $\Delta_{d}$ decrease as $M_{H}^{d}$ increases for $M_{H}^{d} \lesssim 0.6 \mathrm{TeV}$. Note that the constraint from $\Delta M_{B_{d}}$ (130) is stronger than that from $\Delta M_{B_{s}}$ (129). In this region the constraint from $\Delta_{K}$ is not satisfied. But if we relax the constraint (because nonperturbative contributions to $\Delta_{K}$ suffer from large uncertainties) to $\Delta M_{K}^{\text {EXTRA }}<2 \Delta M_{K}^{\exp }$, then it is satisfied.

Next we consider the region in which all the three constraints (117), (129) and (130) are satisfied. We find that the small $M_{H}^{d}$ region in Fig. 4 and 5 disappears, and that $M_{H}^{d} \gtrsim 1.0$ (I) and 1.3 (II) $\mathrm{TeV}$ have to be satisfied. In Fig. 7 and 8 we show the allowed region in which all the constraints (117), (129) and (130) are satisfied for $M_{H}^{d}=1.5 \mathrm{TeV}$ (blue) $M_{H}^{d}=2 \mathrm{TeV}$ (green).

D4: Constraint from $\Delta M_{B_{s}} / \Delta M_{B_{d}}$

This ratio is important to determine experimentally $\left|V_{t d} / V_{t s}\right|$. This is true only if there is no other contribution than the SM ones. In the presence of the extra neutral Higgs bosons, the situation changes. Here we ask ourselves how heavy the extra extra neutral Higgs bosons should be, or where the allowed region in the $r_{d}-\sin \gamma^{d}$ plane for a given $\cos \beta M_{H}^{d}$ is, such that the determination of $\left|V_{t d} / V_{t s}\right|$ from the ratio $\Delta M_{B_{s}} / \Delta M_{B_{d}}$ is not influenced.

The largest theoretical uncertainty in the mass ratio is contained in $\xi=$ 


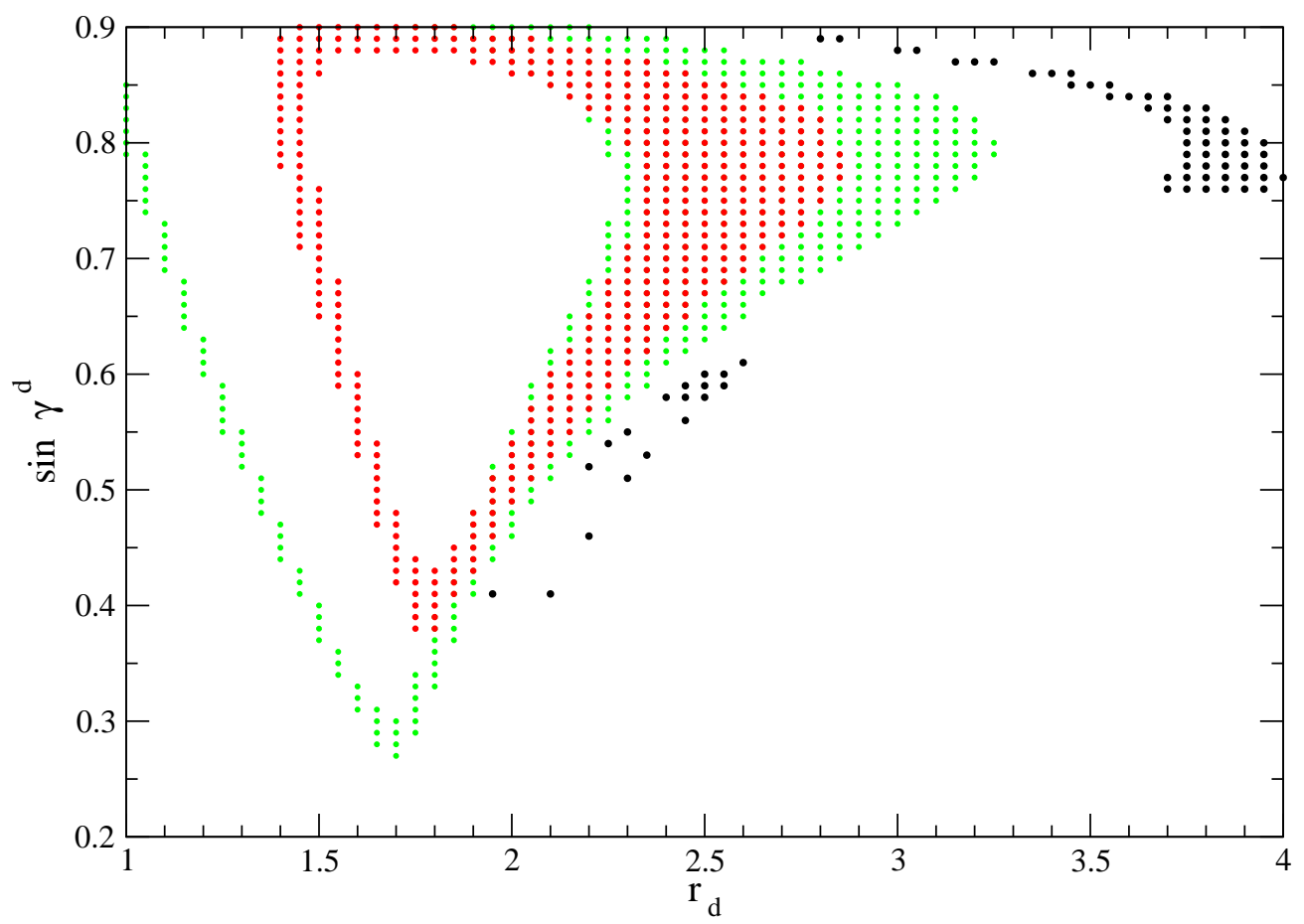

FIG. 9: The allowed region for the parameter set I with $\cos \beta M_{H}^{d}=0.50$ (black), 1.5 (red (dark grey)) and 2 (green (grey)) TeV, in which the constraints (129), (130) and (133) coming from $\Delta M_{B_{s, d}}$ and $\Delta M_{B_{s}} / \Delta M_{B_{d}}$ are simultaneously satisfied.

$f_{B_{s}} \sqrt{B_{s}} / f_{B_{d}} \sqrt{B_{d}}=1.24 \pm 0.04$ (see Table III), that is, $3.2 \%$ uncertainty, which is lager than the experimental ones. Accordingly, we require that the theoretical value of $\Delta M_{B_{s}} / \Delta M_{B_{d}}$ should be equal to the experimental central value 35.05 within an error of $5 \%$ (the mass ratio is proportional to $\xi^{2}$ ), i.e.

$$
\Delta M_{B_{s}} / \Delta M_{B_{d}}=35.05(1 \pm 0.05) .
$$

We require that (129), (130) and (133) are simultaneously satisfied. The allowed region is shown in Fig. 9 and 10 for $M_{H}^{d}=0.50$ (red), 1.5 (blue) and 2 (green) TeV. We see that the small $M_{H}^{d}$ region of Fig. 4 and 5 is still there. We also find that $M_{H}^{d} \gtrsim 1.1$ (1.3) for the parameter set I (II) $\mathrm{TeV}$ or $0.39 \mathrm{TeV} \lesssim M_{H}^{d} \lesssim 0.65\left(0.45 \mathrm{TeV} \lesssim M_{H}^{d} \lesssim 0.6\right) \mathrm{TeV}$ for the parameter set I (II) if $\Delta M_{B_{s}} / \Delta M_{B_{d}}$ is equal to the experimental central value 35.05 within an error of $1 \%$.

\section{CONCLUSION}

We have considered a supersymmetric extension of the SM based on the discrete $Q_{6}$ family symmetry, which has been recently proposed in Refs. [7, 21, 22]. We have stressed the one + two structure for each family; one $Q_{6}$ singlet and one $Q_{6}$ doublet for each family including the $S U(2)_{L}$ doublet Higgs fields. We have found that it is possible to realize the 


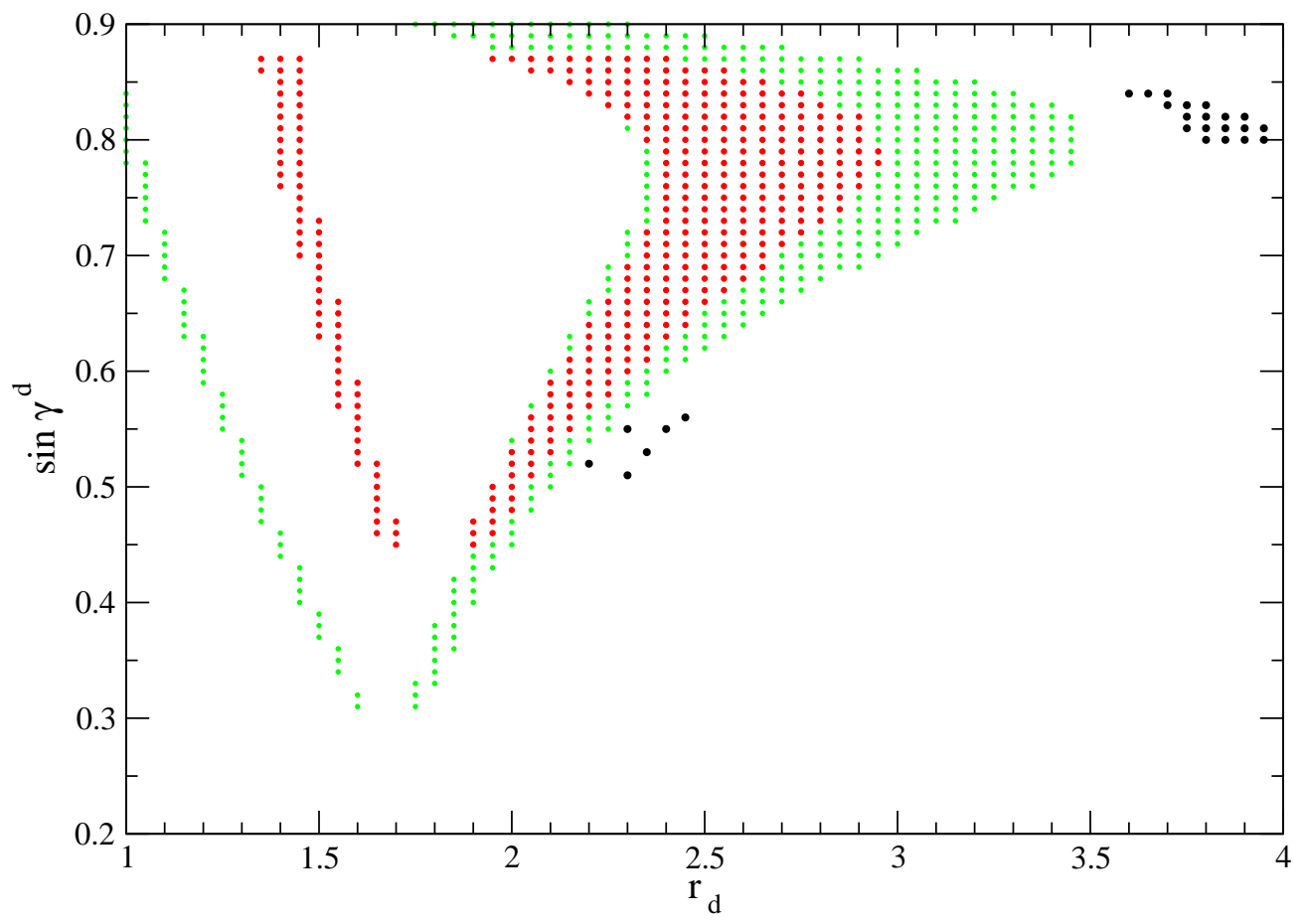

FIG. 10: The same as Fig. 9 for the parameter set II. Two set of values I and II are given in Table III]

one + two structure in a renormalizable way, so that the Higgs sector becomes minimal and much simpler than that of the original model of [7, 21, 22]. In this way the Higgs sector can be investigated with much less assumptions. It is explicitly shown that the SSB parameters can be fine tuned so as to make the heavy Higgs bosons much heavier than $M_{Z}$ and at the same time to obtain a desired size of spontaneous $\mathrm{CP}$ violation to reproduce the Kobayashi-Maskawa CP violating phase.

We have investigated the FCNC processes, especially those mediated by heavy neutral Higgs bosons. Because of the $Q_{6}$ family symmetry, the number of the independent Yukawa couplings is smaller than that of the observed quantities such as the CKM matrix and the quark masses. Therefore, the FCNCs can be parametrized only by the mixing angles and masses of the Higgs fields: There are two angels and four mass parameters that enter into the FCNCs for a given $\tan \beta$; a set of three parameters for $\Delta M_{D}$ and another set of three parameters for $\Delta M_{K}$ and $\Delta M_{B_{d, s}}$. We have expressed the mass differences of the neutral mesons $\Delta M_{K}, \Delta M_{D}$ and $\Delta M_{B_{d, s}}$ in terms of these parameters.

Since the SM contributions to $\Delta M_{B_{s}}$ and $\Delta M_{B_{d}}$ are well-controlled, we haven taken into account them to obtain the constraints from $\Delta M_{B_{s}}$ and $\Delta M_{B_{d}}$. That is, we have assumed that the extra contributions are allowed only in a small window in which the SM values differ from the experimental values. Allowed ranges in which the constraints are satisfied are shown in various figures, where $\Delta M_{K}, \Delta M_{B_{s}}$ and $\Delta M_{B_{d}}$ take values in the common parameter space. We have also investigated the ratio $\Delta M_{B_{s}} / \Delta M_{B_{d}}$ in the region, in which all the constraints from $\Delta M_{B_{s}}$ and $\Delta M_{B_{d}}$ are simultaneously satisfied, and found that in 
a wide subregion the ratio differs from the experimental central value only by less than $5 \%$. If we require that all the constraints from $\Delta M_{K}, \Delta M_{B_{s}}$ and $\Delta M_{B_{d}}$ including the ratio $\Delta M_{B_{s}} / \Delta M_{B_{d}}$ are satisfied, we have found that the heavy Higgs bosons should be heavier than $\sim 1.5 \mathrm{TeV}$. If we relax the constraint from $\Delta M_{K}$ to $\Delta M_{K}^{\text {EXTRA }}<2 \Delta M_{K}^{\text {exp }}$ (because of the reason that non-perturbative contributions suffer from large uncertainties), the heavy Higgs bosons can be as light as $\sim 0.4 \mathrm{TeV}$, which is within the accessible range of LHC [5].

\section{Acknowledgments}

We would like to thank K. Babu, Y. Kajiyama, H. Okada and D. Suematsu for useful discussions. This work is supported by the Grants-in-Aid for Scientific Research from the Japan Society for the Promotion of Science (\# 18540257).

[1] G. Altarelli, Lectures given at the Summer Institute 2007, Fuji-Yoshida, Japan, 3 -10 Aug. 2007, and arXiv:0711.0161 [hep-ph].

[2] E. Ma, arXiv:0705.0327 [hep-ph].

[3] A. Mondragon, AIP Conf. Proc. 857B (2006) 266 arXiv:hep-ph/0609243.

[4] Y. Koide, Phys. Rev. D 71 (2005) 016010 [arXiv:hep-ph/0406286].

[5] C. Buttar et al., arXiv:hep-ph/0604120.

[6] N. Marinelli, arXiv:0709.2335 [hep-ex].

[7] K. S. Babu and J. Kubo, Phys. Rev. D 71 (2005) 056006 arXiv:hep-ph/0411226.

[8] P. H. Frampton and T. W. Kephart, Int. J. Mod. Phys. A 10 (1995) 4689 arXiv:hep-ph/9409330.

[9] P. H. Frampton and T. W. Kephart, Phys. Rev. D 64 (2001) 086007 arXiv:hep-th/0011186.

[10] G. Seidl, arXiv:hep-ph/0301044;

[11] K. R. S. Balaji, M. Lindner and G. Seidl, Phys. Rev. Lett. 91 (2003) 161803 arXiv:hep-ph/0303245.

[12] W. Grimus and L. Lavoura, Phys. Lett. B 572 (2003) 189 [arXiv:hep-ph/0305046].

[13] W. Grimus, A. S. Joshipura, S. Kaneko, L. Lavoura and M. Tanimoto, JHEP 0407 (2004) 078 arXiv:hep-ph/0407112.

[14] C. Hagedorn and W. Rodejohann, JHEP 0507 (2005) 034 arXiv:hep-ph/0503143].

[15] C. Hagedorn, M. Lindner and F. Plentinger, Phys. Rev. D $\mathbf{7 4}$ (2006) 025007 arXiv:hep-ph/0604265.

[16] Y. Kajiyama, J. Kubo and H. Okada, Phys. Rev. D 75 (2007) 033001 arXiv:hep-ph/0610072.

[17] S. L. Chen and E. Ma, Phys. Lett. B 620 (2005) 151 arXiv:hep-ph/0505064.

[18] M. Frigerio, S. Kaneko, E. Ma and M. Tanimoto, Phys. Rev. D 71 (2005) 011901 arXiv:hep-ph/0409187.

[19] S. Okubo, Phys. Rev. D 12 (1975) 3835. 
[20] A. Blum, C. Hagedorn and M. Lindner, arXiv:0709.3450 [hep-ph].

[21] J. Kubo, Phys. Lett. B 622 (2005) 303 arXiv:hep-ph/0506043.

[22] E. Itou, Y. Kajiyama and J. Kubo, Nucl. Phys. B 743 (2006) 74 arXiv:hep-ph/0511268.

[23] J. Kubo, A. Mondragon, M. Mondragon and E. Rodriguez-Jauregui, Prog. Theor. Phys. 109 (2003) 795 [Erratum-ibid. 114 (2005) 287] arXiv:hep-ph/0302196].

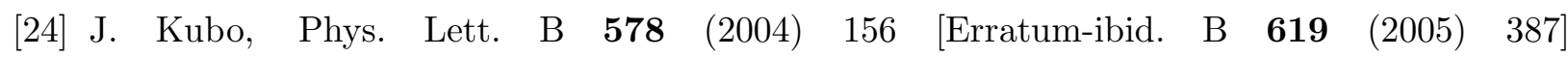
arXiv:hep-ph/0309167.

[25] A. Mondragon, M. Mondragon and E. Peinado, Phys. Rev. D 76 (2007) 076003 arXiv:0706.0354 [hep-ph]].

[26] F. Gabbiani, E. Gabrielli, A. Masiero and L. Silvestrini, Nucl. Phys. B 477 (1996) 321 arXiv:hep-ph/9604387.

[27] M. Ciuchini, E. Franco, A. Masiero and L. Silvestrini, Phys. Rev. D 67 (2003) 075016 [Erratum-ibid. D 68 (2003) 079901] arXiv:hep-ph/0212397].

[28] L. Silvestrini, Int. J. Mod. Phys. A 21 (2006) 1738 arXiv:hep-ph/0510077.

[29] M. Ciuchini and L. Silvestrini, Phys. Rev. Lett. 97 (2006) 021803 arXiv:hep-ph/0603114.

[30] M. Ciuchini, E. Franco, D. Guadagnoli, V. Lubicz, M. Pierini, V. Porretti and L. Silvestrini, arXiv:hep-ph/0703204.

[31] E. Golowich, J. Hewett, S. Pakvasa and A. A. Petrov, arXiv:0705.3650 [hep-ph].

[32] A. Lenz, Phys. Rev. D 76 (2007) 065006 [arXiv:0707.1535 [hep-ph]].

[33] Y. Yamada, Phys. Rev. D 50 (1994) 3537 arXiv:hep-ph/9401241.

[34] J. Hisano and M. A. Shifman, Phys. Rev. D 56 (1997) 5475 arXiv:hep-ph/9705417.

[35] I. Jack and D. R. T. Jones, Phys. Lett. B 415 (1997) 383 arXiv:hep-ph/9709364.

[36] L. V. Avdeev, D. I. Kazakov and I. N. Kondrashuk, Nucl. Phys. B 510 (1998) 289 arXiv:hep-ph/9709397.

[37] I. Jack, D. R. T. Jones and A. Pickering, Phys. Lett. B 426 (1998) 73 arXiv:hep-ph/9712542.

[38] T. Kobayashi, J. Kubo and G. Zoupanos, Phys. Lett. B 427 (1998) 291 arXiv:hep-ph/9802267.

[39] I. Jack, D. R. T. Jones and A. Pickering, Phys. Lett. B 432 (1998) 114 arXiv:hep-ph/9803405.

[40] D. I. Kazakov and V. N. Velizhanin, Phys. Lett. B 485 (2000) 393 arXiv:hep-ph/0005185.

[41] E. Kraus and D. Stockinger, Phys. Rev. D 65 (2002) 105014 arXiv:hep-ph/0201247.

[42] V. A. Novikov, M. A. Shifman, A. I. Vainshtein and V. I. Zakharov, Nucl. Phys. B 229 (1983) 381; Phys. Lett. B 166 (1986) 329.

[43] N. Maekawa, Phys. Lett. B 282 (1992) 387.

[44] J. R. Espinosa, J. M. Moreno and M. Quiros, Phys. Lett. B 319 (1993) 505 arXiv:hep-ph/9308315.

[45] K. S. Babu and S. M. Barr, Phys. Rev. D 49 (1994) 2156 arXiv:hep-ph/9308217.

[46] M. Masip and A. Rasin, Phys. Rev. D 52 (1995) 3768 arXiv:hep-ph/9506471]; Nucl. Phys. B 460 (1996) 449 arXiv:hep-ph/9508365. 
[47] N. Haba, M. Matsuda and M. Tanimoto, Phys. Rev. D 54 (1996) 6928 arXiv:hep-ph/9512421.

[48] S. W. Ham, S. K. Oh and H. S. Song, Phys. Rev. D 61 (2000) 055010 arXiv:hep-ph/9910461. bibitemBranco:2000dq G. C. Branco, F. Kruger, J. C. Romao and A. M. Teixeira, JHEP 0107 (2001) 027 arXiv:hep-ph/0012318.

[49] C. Hugonie, J. C. Romao and A. M. Teixeira, JHEP 0306 (2003) 020 arXiv:hep-ph/0304116.

[50] M. Maniatis, A. von Manteuffel and O. Nachtmann, arXiv:0707.3344 [hep-ph].

[51] W. M. Yao et al. [Particle Data Group], J. Phys. G 33 (2006) 1.

[52] M. Bona et al. [UTfit Collaboration], JHEP 0610 (2006) 081.

[53] H. D. Kim, S. Raby and L. Schradin, Phys. Rev. D 69 (2004) 092002 arXiv:hep-ph/0401169].

[54] O. Felix, A. Mondragon, M. Mondragon and E. Peinado, AIP Conf. Proc. 917 (2007) 383 [Rev. Mex. Fis. S52N4 (2006) 67] arXiv:hep-ph/0610061].

[55] Y. Kajiyama, JHEP 0704 (2007) 007 arXiv:hep-ph/0702056.

[56] L. J. Hall, V. A. Kostelecky and S. Raby, Nucl. Phys. B 267 (1986) 415.

[57] F. Gabbiani and A. Masiero, Nucl. Phys. B 322 (1989) 235.

[58] S. Abel, S. Khalil and O. Lebedev, Nucl. Phys. B 606 (2001) 151 arXiv:hep-ph/0103320.

[59] M. Endo, M. Kakizaki and M. Yamaguchi, Phys. Lett. B $\mathbf{5 8 3}$ (2004) 186 arXiv:hep-ph/0311072.

[60] J. Hisano, Nucl. Phys. Proc. Suppl. 137 (2004) 169 arXiv:hep-ph/0405185.

[61] J. Hisano and Y. Shimizu, Phys. Rev. D 70 (2004) 093001 arXiv:hep-ph/0406091.

[62] W. Grimus, L. Lavoura, O. M. Ogreid and P. Osland, arXiv:0711.4022 [hep-ph].

[63] G. Buchalla, A. J. Buras and M. E. Lautenbacher, Rev. Mod. Phys. 68 (1996) 1125 arXiv:hep-ph/9512380.

[64] M. Ciuchini, E. Franco, V. Lubicz, G. Martinelli, I. Scimemi and L. Silvestrini, Nucl. Phys. B 523 (1998) 501 arXiv:hep-ph/9711402.

[65] M. Artuso et al. [CLEO Collaboration], Phys. Rev. Lett. 95 (2005) 251801 arXiv:hep-ex/0508057.

[66] D. Asner et. al. [Heavy Flavor Averaging Group], http://www.slac.stanford.edu/xorg/hfag/charm/index.html

[67] E. Dalgic et al., Phys. Rev. D 76 (2007) 011501 arXiv:hep-lat/0610104.

[68] N. Tantalo, arXiv:hep-ph/0703241.

[69] M. Della Morte, PoS LAT2007 (2007) 008 [arXiv:0711.3160 [hep-lat]].

[70] D. Becirevic, V. Gimenez, G. Martinelli, M. Papinutto and J. Reyes, JHEP 0204 (2002) 025 arXiv:hep-lat/0110091].

[71] A. Abulencia et al. [CDF Collaboration], Phys. Rev. Lett. 97 (2006) 211802 arXiv:hep-ex/0607021.

[72] A. Lenz and U. Nierste, JHEP 0706 (2007) 072 [arXiv:hep-ph/0612167].

[73] Y. Aoki et al., Phys. Rev. D 73 (2006) 094507 [arXiv:hep-lat/0508011].

[74] D. Becirevic and G. Villadoro, Phys. Rev. D 70 (2004) 094036 arXiv:hep-lat/0408029].

[75] M. Beneke, G. Buchalla, A. Lenz and U. Nierste, Phys. Lett. B 576 (2003) 173 
arXiv:hep-ph/0307344.

[76] M. Beneke, G. Buchalla, C. Greub, A. Lenz and U. Nierste, Phys. Lett. B 459 (1999) 631 arXiv:hep-ph/9808385. 